\title{
QUADRATIC TRANSFORMATION FORMULAS FOR BASIC HYPERGEOMETRIC SERIES
}

\author{
MIZAN RAHMAN AND ARUN VERMA
}

\begin{abstract}
Starting with some of the known transformation formulas for wellpoised ${ }_{2} \phi_{1}$ and very-well-poised ${ }_{8} \phi_{7}$ basic hypergeometric series we obtain $q$-analogues of 36 quadratic transformation formulas given in $\$ 2.11$ of $\mathrm{Higher}$ transcendental functions, Vol. 1, edited by Erdélyi et al. We also derive some new quadratic transformation formulas that give rise to identities connecting very-well-poised but unbalanced ${ }_{10} \phi_{9}$ series in base $q$ with very-well-poised and balanced ${ }_{12} \phi_{11}$ series in base $q^{2}$. A Rogers-Ramanujan type identity is also found as a limiting case.
\end{abstract}

\section{INTRODUCTION}

It is probably safe to assume that all of us who have worked on classical orthogonal polynomials and special functions or other related areas have, one time or another, made use of Goursat's [22] extensive list of quadratic transformation formulas for Gauss' hypergeometric function $F(a, b ; c ; z)$ that the editors of the Bateman Manuscript Project have so thoughtfully reproduced in [15]. They are extremely useful for students and researchers alike. Their history goes back to Gauss [20,21] himself and also to Kummer [31]. They had been generalized by Whipple $[43,44,45]$ and by Bailey $[9,10,11]$, among others, to higher order hypergeometric functions that were also found useful in many applications. But the generalizations that are most important today, and are crying out to be done as completely as possible, are the so-called $q$-analogues of the ones that we already know. The first glimpse of such work seems to occur in Sear's 1951 paper [38], one of the main results of which also appeared in a later paper by Carlitz [13]. An important quadratic transformation formula for a terminating balanced ${ }_{4} \phi_{3}$ series was given by Singh [39] in 1959 in his attempt at generalizing the Cayley-Orr type identities. Askey and Wilson [8] gave an alternate proof of this formula in 1985 and used it to find different forms of the Askey-Wilson polynomials that have become a focal point of current research in orthogonal polynomials. But, apart from some notable but sporadic results in the 1960s (see, for example, R. P. Agarwal's [1] book of 1963 and L. J. Slater's

Received by the editors August 22, 1990 and, in revised form, October 12, 1990.

1980 Mathematics Subject Classification (1985 Revision). Primary 33A30, 33A65.

Key words and phrases. Basic hypergeometric series, balanced and very-well-posed series, quadratic transformation formulas, Rogers-Ramanujan identities.

This work was supported in part by the NSERC grant \#A6197. 
[40] book of 1966) there did not seem to be any real interest in basic analogues of quadratic summation and transformation formulas (or, for that matter, in basic hypergeometric series as a separate branch of mathematics) until some papers of Andrews [3, 4, 5] and later of Andrews and Askey [6, 7] began to appear in the 1970s and early eighties. All of a sudden basic hypergeometric series became a fashionable thing to do-the " $q$-disease" began to spread. We saw a fairly large number of papers appearing in reputable journals all over the world on $q$-series and their applications. Naturally, there was also a renewed interest in the $q$-analogues of quadratic transformations. Useful works were done in the early eighties by Jain [23, 24], by Jain and Verma [25, 26, 27], Nassrallah [34], Nassrallah and Rahman [35], Al-Salam and Verma [2], Gasper [16], Gasper and Rahman [17, 18], and Rahman [36]. The reader will find a gist of much of this work in Gasper and Rahman's [19] recent book which contains the ingredients of the basic analogues of all the formulas of Goursat that are listed in $\S 2.11$ of [15].

The basic work of Drinfel'd [14] has spurred tremendous interest in quantum groups and their representations in recent years. This, in turn, has created an interest among group-theorists in basic hypergeometric series who would probably not otherwise have much to do with them. Although the connection between special functions and group representation theory is well known (see, for example, Vilenkin's [42] book), group-theoretic interpretations and derivations of many of the nice results in basic hypergeometric orthogonal polynomials have eluded the harmonic analysts until Woronowicz [46, 47], Vaksman and Soibl'man [41], Masuda et al. [32], Mimachi and Noumi [33], Koornwinder $[28,29]$ and others found connections between basic hypergeometric series and the representations of $S U_{q}(2)$ groups. In his most recent work Koornwinder [30], motivated by the quantum group-theoretic approach, obtained a second addition formula for Rogers' $q$-ultraspherical polynomials, besides the earlier one by Rahman and Verma [37], and there he felt the need for $q$-analogues of Gauss-Kummer quadratic transformation formulas. He found Gasper and Rahman's book very useful but lamented to one of us (MR) at the 1990 meeting on Orthogonal Polynomials in Erice, Italy, the absence of an explicitly displayed $q$-analogue of Goursat's list. The principal motivation of this paper came from that discussion with Tom Koornwinder.

In $\S 2$ we shall introduce the notations and definitions as well as the formulas that will form the basis for all the quadratic transformation formulas that are $q$ analogues of Goursat's list. These formulas are, of course, available in [19], but the reader will be able to follow the proofs if all the tools are displayed together. In $\S \S 3-6$ we will present four groups of quadratic transformations such that there is a structural similarity among the transformations in each group. The formulas presented in these four sections will together be a comprehensive list of the $q$-analogues of all the formulas given in $[15,2.11]$. There may be other $q$-analogues, of course, and in fact for some of them we know the existence of others, but we believe that the ones presented here are the more useful ones, at least as far as their applications to orthogonal polynomials are concerned.

In $\S 7$ we shall also present some quadratic transformation formulas which we believe to be new and which has among its special and limiting cases some useful formulas given in [19]. 


\section{DEFINITIONS AND SOME BASIC FORMULAS}

Following [19] we shall define an ${ }_{r} \phi_{s}$ basic hypergeometric series by

$$
\begin{aligned}
{ }_{r} \phi_{s} & \left(a_{1}, a_{2}, \ldots, a_{r} ; b_{1}, b_{2}, \ldots, b_{s} ; q, z\right) \\
& \equiv{ }_{r} \phi_{s}\left[\begin{array}{c}
a_{1}, \ldots, a_{r} \\
b_{1}, b_{2}, \ldots, b_{s} ; q, z
\end{array}\right] \\
& :=\sum_{n=0}^{\infty} \frac{\left(a_{1}, a_{2}, \ldots, a_{r} ; q\right)_{n}}{\left(q, b_{1}, \ldots, b_{s} ; q\right)_{n}}\left[(-1)^{n} q^{n(n-1) / 2}\right]^{1+s-r} z^{n},
\end{aligned}
$$

where $q$ is a fixed complex number called the base of the series, and $z$ is a complex argument. The $q$-shifted factorials are defined as follows:

$$
\begin{aligned}
& (a ; q)_{n}=\left\{\begin{array}{l}
1 \quad \text { if } n=0 \\
(1-a)(1-a q) \cdots\left(1-a q^{n-1}\right), \quad n=1,2, \ldots,
\end{array}\right. \\
& (a ; q)_{\infty}=\lim _{n \rightarrow \infty}(a ; q)_{n}, \quad|q|<1, \\
& \left(a_{1}, a_{2}, \ldots, a_{r} ; q\right)_{n}=\prod_{j=1}^{r}\left(a_{j} ; q\right)_{n} .
\end{aligned}
$$

If any one of the $r$ numerator parameters in (2.1) has the form $q^{-m}, m=$ $0,1,2, \ldots$, then the series terminates provided, of course, that there are no zero factors in the denominator. Otherwise it is an infinite series which converges for certain restricted values of $q, z, r, s$, and the parameters $a$ 's and $b$ 's. For a complete discussion of the conditions of convergence see [19]. For the purpose of this paper we shall restrict $q$ to $(0,1), z$ to $|z|<1$, and $r, s$ to $r \leq s+1$. For a complex number $c$ we shall also use the notation

$$
(a ; q)_{c}=(a ; q)_{\infty} /\left(a q^{c} ; q\right)_{\infty}, \quad\left|a q^{c}\right|<1,
$$

which is a $q$-analogue of $(1-a)^{c},|a|<1$, via the $q$-binomial formula [19, (1.3.2)]

$$
{ }_{1} \phi_{0}(a ;-; q, z)=(a z ; q)_{\infty} /(z ; q)_{\infty}, \quad|z|<1 .
$$

We shall also need the $q$-gamma function defined by

$$
\begin{gathered}
\Gamma_{q}(x)=\frac{(q ; q)_{\infty}}{\left(q^{x} ; q\right)_{\infty}}(1-q)^{1-x}, \quad x \neq 0,-1,-2, \ldots, 0<q<1, \\
\lim _{q \rightarrow 1^{-}} \Gamma_{q}(x)=\Gamma(x) .
\end{gathered}
$$

An ${ }_{r+1} \phi_{r}$ series is called balanced (or Saalschützian) if $z=q$ and $b_{1} b_{2} \cdots b_{r}$ $=q a_{1} a_{2} \cdots a_{r+1}$. An ${ }_{r+1} \phi_{r}$ series is called well-poised if the paramaters satisfy the relations $q a_{1}=a_{2} b_{1}=\cdots=a_{r+1} b_{r}$; very-well-poised if, in addition, $a_{2}=q a_{1}^{\frac{1}{2}}, a_{3}=-q a_{1}^{\frac{1}{2}}$. It is called a nearly-poised series of the first kind if $q a_{1} \neq a_{2} b_{1}=a_{3} b_{2}=\cdots=a_{r+1} b_{r}$, and a nearly-poised series of the second kind if $q a_{1}=a_{2} b_{1}=\cdots=a_{r} b_{r-1} \neq a_{r+1} b_{r}$. Balanced, wellpoised and, particularly, very-well-poised series will feature very prominently in this paper. For printing economy we shall sometimes use the notation ${ }_{r+1} W_{r}\left(a_{1} ; a_{4}, a_{5}, \ldots, a_{r+1} ; q, z\right)$ for the very-well-poised series

$$
{ }_{r+1} \phi_{r}\left(a_{1}, q a_{1}^{\frac{1}{2}},-q a_{1}^{\frac{1}{2}}, a_{4}, \ldots, a_{r+1} ; a_{1}^{\frac{1}{2}}-a_{1}^{\frac{1}{2}}, q a_{1} / a_{4}, \ldots, q a_{1} / a_{r+1} ; q, z\right) \text {. }
$$


However, in $\S \S 3-6$ where explicit displays of both numerators and denominator parameters of very-well-poised ${ }_{8} \phi_{7}$ series will be deemed necessary we shall replace $q \sqrt{a_{1}},-q \sqrt{a_{1}}$ in the numerator, and $\sqrt{a_{1}},-\sqrt{a_{1}}$ in the denominator by $q \sqrt{\cdot},-q \sqrt{\cdot}$ and $\sqrt{\cdot},-\sqrt{\cdot}$, respectively, the dot being whatever happens to be the leading element $a_{1}$ in the given series.

The quadratic transformation formulas that we shall present in $\S \S 3-6$ originate, in one form or another, from the following two formulas:

$$
\begin{aligned}
& { }_{2} \phi_{1}\left(a, b ; a q / b ; q, q x / b^{2}\right)=\frac{\left(x q / b, a q x^{2} / b^{2} ; q\right)_{\infty}}{\left(a x q / b, q x^{2} / b^{2} ; q\right)_{\infty}} \\
& \cdot{ }_{8} W_{7}\left(a x / b ; x, a^{\frac{1}{2}},-a^{\frac{1}{2}},(a q)^{\frac{1}{2}},-(a q)^{\frac{1}{2}} ; q, q x / b^{2}\right),
\end{aligned}
$$

provided $\left|q x / b^{2}\right|<1$ when the series do not terminate, and

$$
\begin{aligned}
{ }_{2} \phi_{1}\left(a^{2}, b^{2} ; q^{2} a^{2} / b^{2} ; q^{2}, q^{2} x^{2} / b^{4}\right) \\
=\frac{\left(q a^{2} x^{2} / b^{2}, q^{2} a^{2} x^{2} / b^{4} ; q^{2}\right)_{\infty}\left(-x q^{\frac{1}{2}} / b,-a x q^{\frac{3}{2}} / b^{3} ; q\right)_{\infty}}{\left(q x^{2} / b^{2}, q^{2} x^{2} / b^{4} ; q^{2}\right)_{\infty}\left(-a x q^{\frac{1}{2}} / b,-a^{2} x q^{\frac{3}{2}} / b^{3} ; q\right)_{\infty}} \\
\quad{ }_{8} W_{7}\left(-\frac{x a^{2} q^{\frac{1}{2}}}{b^{3}} ; a, \frac{a q^{\frac{1}{2}}}{b},-\frac{a q^{\frac{1}{2}}}{b},-\frac{q a}{b^{2}},-\frac{x q^{\frac{1}{2}}}{b} ; q,-\frac{x q^{\frac{1}{2}}}{b}\right),
\end{aligned}
$$

provided $\left|q x / b^{2}\right|<1$ and and $\left|x q^{\frac{1}{2}} / b\right|<1$ when the series do not terminate. The above two formulas are, respectively, (3.4.7) and (3.5.5) of [19]. By combining (2.8) and (2.9) one may obtain a transformation formula between two very-well-poised ${ }_{8} \phi_{7}$ series in bases $q$ and $q^{2}$, namely,

$$
\begin{aligned}
{ }_{8} W_{7}\left(a ; b, c, c q,-d,-d q ; q^{2}, a^{2} q^{2} / b c^{2} d^{2}\right) \\
=\frac{\left(a^{2} q^{2} / c^{2} d^{2}, a^{2} q^{2} / b d^{2} ; q^{2}\right)_{\infty}(a q, a q / b c ; q)_{\infty}}{\left(a^{2} q^{2} / d^{2}, a^{2} q^{2} / b c^{2} d^{2} ; q^{2}\right)_{\infty}(a q / b, a q / c ; q)_{\infty}} \\
\quad \cdot{ }_{8} W_{7}\left(\frac{a}{d} ; c, b^{\frac{1}{2}},-b^{\frac{1}{2}}, \frac{(a q)^{\frac{1}{2}}}{d},-\frac{(a q)^{\frac{1}{2}}}{d} ; q, \frac{a q}{b c}\right),
\end{aligned}
$$

where $\left|a^{2} q^{2} / b c^{2} d^{2}\right|<1,|a q / b c|<1$ when the series do not terminate, see $[19,(3.5 .10)]$.

In order to have a complete list of $q$-analogues of Goursat's formulas we shall also need the following linear transformations:

$$
{ }_{2} \phi_{1}(a, b ; c ; q, z)=\frac{(a z ; q)_{\infty}}{(z ; q)_{\infty}}{ }_{2} \phi_{2}(a, c / b ; c, a z ; q, b z),
$$

$|z|<1$, see $[19,(1.5 .4)]$, and

$$
\begin{aligned}
{ }_{8} W_{7} & (a ; b, c, d, e, f ; q, \lambda q / e f) \\
= & \frac{(a q, a q / e f, \lambda q / e, \lambda q / f ; q)_{\infty}}{(a q / e, a q / f, \lambda q, \lambda q / e f ; q)_{\infty}} \\
& \cdot{ }_{8} W_{7}(\lambda ; \lambda b / a, \lambda c / a, \lambda d / a, e, f ; q, a q / e f),
\end{aligned}
$$

where $\lambda=q a^{2} / b c d$, and $\max (|a q / e f|,|\lambda q / e f|)<1$ if the series do not terminate, see [19, (2.10.1)]. Both (2.11) and (2.12) may be regarded as $q$-analogues of Pfaff-Kummer transformation formulas

$$
F(a, b ; c ; z)=(1-z)^{-a} F(a, c-b ; c ; z /(z-1)) .
$$


Later, there will be need for using two further, somewhat more complicated, transformation formulas which we shall state in the relevant sections.

For the piece of work that we shall present in $\S 7$ the most basic formula is Andrews' [5] $q$-analogue of Watson's summation formula [11, 3.3(1)]:

$$
\begin{aligned}
{ }_{4} \phi_{3}\left[\begin{array}{c}
q^{-n}, a^{2} q^{n}, b,-b \\
a \sqrt{q},-a \sqrt{q}, b^{2} ; q, q
\end{array}\right] \\
\quad= \begin{cases}0 & \text { if } n \text { is odd, } \\
\frac{\left(q, a^{2} q / b^{2} ; q^{2}\right)_{\frac{n}{2}} b^{n}}{\left(q a^{2}, q b^{2} ; q^{2}\right)_{\frac{n}{2}}}, & \text { if } n \text { is even, }\end{cases}
\end{aligned}
$$

$n=0,1,2, \ldots$, which is really a variation of the $q$-Dixon formula [19, (2.7.2)] via Watson's transformation formula [19, (2.5.1)].

\section{QUADRATIC TRANSFORMATION FORMULAS. I}

In this and the three following sections we shall present the main formulas in a form so that their limits as $q \rightarrow 1^{-}$can be evaluated directly. Although the limits of the series will exist term-by-term, no attempt will be made to give a rigorous justification of these limiting processes. In each instance, however, we shall state the conditions of convergence with the understanding that they are not necessary when the series terminate. By involving a dominant convergence theorem it is not difficult to justify the $q \rightarrow 1^{-}$limiting process in each formula.

If we replace $a, b$ in (2.8) by $q^{a}, q^{b}$, respectively, then we obtain

$$
\begin{aligned}
& { }_{2} \phi_{1}\left(q^{a}, q^{b} ; q^{1+a-b} ; q, x q^{1-2 b}\right) \\
& =\frac{\left(x q^{1-b} ; q\right)_{a}}{\left(x^{2} q^{1-2 b} ; q\right)_{a}} \\
& \quad \cdot{ }_{8} \phi_{7}\left[\begin{array}{c}
x q^{a-b}, q \sqrt{\cdot},-q \sqrt{\cdot}, q^{\frac{a}{2}}, q^{\frac{a+1}{2}}, \\
\sqrt{\cdot},-\sqrt{\cdot}, x q^{\frac{2+a-2 b}{2}}, x q^{\frac{1+a-2 b}{2}}, \\
\quad-q^{\frac{a}{2}},-q^{\frac{a+1}{2}}, x \\
\quad-x q^{\frac{2+a-2 b}{2}},-x q^{\frac{1+a-2 b}{2}}, q^{1+a-b}
\end{array} ; q, x q^{1-2 b}\right],
\end{aligned}
$$

$\left|x q^{1-2 b}\right|<1$. The $q \rightarrow 1^{-}$limit of this formula is

$$
\begin{aligned}
& F(a, b ; 1+a-b ; x) \\
& \quad=(1+x)^{-a} F\left(\frac{a}{2}, \frac{a+1}{2} ; 1+a-b ; 4 x(1+x)^{-2}\right),
\end{aligned}
$$

which is $[15,2.11(34)]$. Applying $(2.10)$ to the ${ }_{8} \phi_{7}$ series in (3.1) we get

$$
\begin{gathered}
{ }_{2} \phi_{1}\left(q^{a}, q^{b} ; q^{1+a-b} ; q, x q^{1-2 b}\right) \\
=\frac{\left(x q^{\frac{1}{2}-b} ; q\right)_{1+a-b}\left(-x q^{1+\frac{a}{2}-2 b},-x q^{\frac{3}{2}+\frac{a}{2}-2 b} ; q\right)_{b-\frac{1}{2}}}{\left(x q^{1-2 b} ; q\right)_{b}\left(x^{2} q^{1-2 b} ; q\right)_{a}} \\
\cdot{ }_{8} \phi_{7}\left[\begin{array}{c}
x q^{\frac{1}{2}+a-2 b}, q \sqrt{\cdot},-q \sqrt{\cdot}, q^{\frac{a+1-2 b}{2}}, q^{\frac{a+2-2 b}{2}},-q^{\frac{a}{2}}, \\
\sqrt{\cdot},-\sqrt{\cdot}, x q^{\frac{2+a-2 b}{2}}, x q^{\frac{1+a-2 b}{2}},-x q^{\frac{3+a}{2}-2 b}, \\
-q^{\frac{a+1}{2}}, x q^{\frac{1}{2}-b} \\
\left.-x q^{\frac{2+a}{2}-2 b}, q^{1+a-b} ; q, x q^{\frac{1}{2}-b}\right],
\end{array}\right.
\end{gathered}
$$


$\left|x q^{1-2 b}\right|<1$, which is a $q$-analogue of [15, 2.11(35)]:

$$
\begin{aligned}
& F(a, b ; 1+a-b ; x)=(1-x)^{1-2 b}(1+x)^{2 b-a-1} \\
& \quad \cdot F\left(\frac{a+1}{2}-b, \frac{a+2}{2}-b ; 1+a-b ; 4 x(1+x)^{-2}\right) .
\end{aligned}
$$

Taking other combinations of parameters in applying (2.10) to the ${ }_{8} \phi_{7}$ series in (3.1) we obtain two additional formulas:

$$
\begin{aligned}
& { }_{2} \phi_{1}\left(q^{a}, q^{b} ; q^{1+a-b} ; q, x q^{1-2 b}\right) \\
& =\frac{\left(x q^{1+\frac{a}{2}-2 b} ; q\right)_{b}\left(-x q^{1-b},-x q^{1+\frac{a}{2}-2 b} ; q\right)_{\frac{a}{2}}}{\left(x q^{1-2 b} ; q\right)_{b}\left(x^{2} q^{1-2 b} ; q\right)_{a}} \\
& \cdot{ }_{8} \phi_{7}\left[\begin{array}{l}
-x q^{a-2 b}, q \sqrt{\cdot},-q \sqrt{\cdot}, q^{\frac{a}{2}}, q^{\frac{a+1}{2}-b}, \\
\sqrt{\cdot},-\sqrt{\cdot},-x q^{\frac{2+a}{2}-2 b},-x q^{\frac{1+a}{2}-2 b},
\end{array}\right. \\
& \begin{array}{c}
-q^{\frac{a}{2}},-q^{\frac{a+1}{2}-b},-x q^{-b} \\
\left.x q^{\frac{2+a}{2}-b}, x q^{\frac{1+a}{2}-b}, q^{1+a-b} ; q,-x q^{1-b}\right],
\end{array}
\end{aligned}
$$

$\left|x q^{1-2 b}\right|<1$, and

$$
\begin{aligned}
& { }_{2} \phi_{1}\left(q^{a}, q^{b} ; q^{1+a-b} ; q, x q^{1-2 b}\right) \\
& =\frac{\left(x q^{1-b} ; q\right)_{\frac{a-1}{2}}\left(-x q^{-b},-x q^{\frac{3+a}{2}-2 b} ; q\right)_{\frac{a+1}{2}}}{\left(x q^{1-2 b} ; q\right)_{\frac{a+1}{2}}\left(x^{2} q^{1-2 b} ; q\right)_{a}} \\
& { }_{8} W_{7}\left[\begin{array}{l}
-x q^{1+a-2 b}, q \sqrt{\cdot},-q \sqrt{\cdot}, q^{\frac{a+1}{2}}, q^{\frac{a+2}{2}-b},-q^{\frac{a+1}{2}}, \\
\sqrt{\cdot},-\sqrt{\cdot},-x q^{\frac{3+a}{2}-2 b},-x q^{\frac{2+a}{2}-b}, x q^{\frac{3+a}{2}-2 b},
\end{array}\right. \\
& \begin{array}{l}
-q^{\frac{a+2}{2}-b},-x q^{1-b} \\
\left.x q^{\frac{a+2}{2}-b}, q^{1+a-b} ; q,-x q^{-b}\right],
\end{array}
\end{aligned}
$$

$\left|x q^{1-2 b}\right|<1,\left|x q^{-b}\right|<1$, which are $q$-analogues of $[15,2.11(32)]$ :

$$
F(a, b ; 1+a-b ; x)
$$

$$
=(1-x)^{-a} F\left(\frac{a}{2}, \frac{a+1}{2}-b ; 1+a-b ;-4 x(1-x)^{-2}\right)
$$

and $[15,2.11(33)]$ :

$$
\begin{aligned}
& F(a, b ; 1+a-b ; x) \\
& \quad=(1+x)(1-x)^{-a-1} F\left(\frac{a+1}{2}, \frac{a+2}{2}-b ; 1+a-b ;-4 x(1-x)^{-2}\right),
\end{aligned}
$$

respectively. Replacing $a$ and $b$ in (3.6) by $2 a-1$ and $a-b+\frac{1}{2}$, respectively, we obtain

$$
\begin{aligned}
& { }_{8} \phi_{7}\left[\begin{array}{l}
-x q^{2 b-1}, q \sqrt{\cdot},-q \sqrt{\cdot}, q^{a}, q^{b},-q^{a}, \\
\sqrt{\cdot},-\sqrt{\cdot},-x q^{2 b-a},-x q^{b}, x q^{2 b-a},
\end{array}\right. \\
& \left.\begin{array}{l}
-q^{b},-x q^{b-a+\frac{1}{2}} \\
x q^{b}, q^{a+b-\frac{1}{2}}
\end{array} q,-x q^{b-a-\frac{1}{2}}\right] \\
& =\frac{\left(x q^{2 b-2 a} ; q\right)_{a}\left(x^{2} q^{2 b-2 a} ; q\right)_{2 a-1}}{\left(x q^{b-a+\frac{1}{2}} ; q\right)_{a-1}\left(-x q^{b-a-\frac{1}{2}},-x q^{2 b-a} ; q\right)_{a}} \\
& \cdot{ }_{2} \phi_{1}\left(q^{2 a-1}, q^{a-b+\frac{1}{2}} ; q^{a+b-\frac{1}{2}} ; q, x q^{2 b-2 a}\right) \text {, }
\end{aligned}
$$


$\left|x q^{b-a-\frac{1}{2}}\right|<1,\left|x q^{2 b-2 a}\right|<1$, which is a $q$-analogue of [15, 2.11(14)]:

$$
\begin{aligned}
& F\left(a, b ; a+b-\frac{1}{2} ; z\right) \\
& =(1-z)^{-\frac{1}{2}}\left(1+(1-z)^{\frac{1}{2}}\right)^{1-2 a} 2^{2 a-1} \\
& \quad \cdot F\left(2 a-1, a-b+\frac{1}{2} ; a+b-\frac{1}{2} ;\left((1-z)^{\frac{1}{2}}-1\right) /\left((1-z)^{\frac{1}{2}}+1\right)\right) .
\end{aligned}
$$

On the other hand, replacing $a$ and $b$ in (3.5) by $2 a$ and $a-b+\frac{1}{2}$, respectively, we obtain

$$
\begin{aligned}
& { }_{2} \phi_{1}\left(q^{2 a} ; q^{a-b+\frac{1}{2}} ; q^{a+b+\frac{1}{2}} ; q, x q^{2 b-2 a}\right) \\
& =\frac{\left(x q^{b-a+\frac{1}{2}},-x q^{b-a+\frac{1}{2}},-x q^{2 b-a} ; q\right)_{a}}{\left(x q^{2 b-2 a} ; q\right)_{a}\left(x^{2} q^{2 b-2 a} ; q\right)_{2 a}} \\
& \quad{ }_{8} \phi_{7}\left[\begin{array}{c}
-x q^{2 b-1}, q \sqrt{\cdot},-q \sqrt{\cdot}, q^{a}, q^{b},-q^{a-\frac{1}{2}} \\
\sqrt{\cdot},-\sqrt{\cdot},-x q^{2 b-a},-x q^{b}, x q^{2 b-a+\frac{1}{2}}, \\
-q^{b-\frac{1}{2}},-x q^{b-a-\frac{1}{2}}
\end{array} ; q,-x q^{b-a+\frac{1}{2}}\right], \\
& x q^{b+\frac{1}{2}}, q^{a+b+\frac{1}{2}}
\end{aligned}
$$

$\left|x q^{2 b-2 a}\right|<1$, which is a $q$-analogue of $[15,2.11(11)]$ :

$$
\begin{aligned}
F(2 a & \left., a-b+\frac{1}{2} ; a+b+\frac{1}{2} ; x\right) \\
\quad & (1-x)^{-2 a} F\left(a, b ; a+b+\frac{1}{2} ;-4 x(1-x)^{-2}\right) .
\end{aligned}
$$

Replacing $a$ and $b$ in (3.1) by $2 a$ and $2 a-b+1$, respectively, gives

$$
\begin{gathered}
{ }_{8} \phi_{7}\left[\begin{array}{c}
x q^{b-1}, q \sqrt{\cdot},-q \sqrt{\cdot}, q^{a}, q^{a+\frac{1}{2}},-q^{a},-q^{a+\frac{1}{2}}, x \\
\sqrt{\cdot},-\sqrt{\cdot}, x q^{b-a}, x q^{b-a-\frac{1}{2}},-x q^{b-a},-x q^{b-a-\frac{1}{2}}, q^{b} ; q, x q^{2 b-4 a-1}
\end{array}\right] \\
=\frac{\left(x^{2}, q^{2 b-4 a-1} ; q\right)_{2 a}}{\left(x q^{b-2 a} ; q\right)_{2 a}}{ }_{2} \phi_{1}\left(q^{2 a}, q^{2 a-b+1} ; q^{b} ; q, x q^{2 b-4 a-1}\right),
\end{gathered}
$$

$\left|x q^{2 b-4 a-1}\right|<1$, whose $q \rightarrow 1^{-}$limit is

$$
F\left(a, a+\frac{1}{2} ; b ; 4 x(1+x)^{-2}\right)=(1+x)^{2 a} F(2 a, 2 a-b+1 ; b ; x)
$$

that reduces to $[15,2.11(6)]$ with $x$ replaced by $z /(2-z)$.

Use of (2.11) on the ${ }_{2} \phi_{1}$ series in (3.13) gives yet another quadratic transformation

$$
\begin{aligned}
& { }_{8} \phi_{7}\left[\begin{array}{c}
x q^{b-1}, q \sqrt{\cdot},-q \sqrt{\cdot}, q^{a}, q^{a+\frac{1}{2}},-q^{a},-q^{a+\frac{1}{2}}, x \\
\sqrt{\cdot},-\sqrt{\cdot}, x q^{b-a}, x q^{b-a-\frac{1}{2}},-x q^{b-a},-x q^{b-a-\frac{1}{2}}, q^{b}
\end{array} ;, x q^{2 b-4 a-1}\right] \\
& =\frac{\left(x^{2} q^{2 b-4 a-1)} ; q\right)_{2 a}}{\left(x q^{b-2 a}, x q^{2 b-4 a-1} ; q\right)_{2 a}}{ }_{2} \phi_{2}\left(q^{2 a}, q^{2 b-2 a-1} ; q^{b}, x q^{2 b-2 a-1} ; q, x q^{b-2 a}\right), \\
& \left|x q^{2 b-4 a-1}\right|<1, \text { whose } q \rightarrow 1^{-} \text {limit is } \\
& \text { (3.16) } \quad F\left(a, a+\frac{1}{2} ; b ; 4 x(1+x)^{-2}\right) \\
& =\left(\frac{1+x}{1-x}\right)^{2 a} F(2 a, 2 b-2 a-1 ; b ; x /(x-1)),
\end{aligned}
$$


which is the same as $[15,2.11(16)]$ with $(1-\sqrt{1-z}) /(1+\sqrt{1-z})$ replacing $x$.

We now replace $b$ By $(1+a-b) / 2$ in (3.1) and use (2.11) to get

$$
\begin{aligned}
& { }_{2} \phi_{2}\left(q^{a}, q^{b} ; q^{\frac{a+b+1}{2}}, x q^{b} ; q, x q^{\frac{b-a+1}{2}}\right) \\
& =\frac{\left(x q^{b-a}, x q^{\frac{b-a+1}{2}} ; q\right)_{a}}{\left(x^{2} q^{b-a} ; q\right)_{a}} \\
& \qquad \begin{array}{r}
{ }_{8} \phi_{7}\left[\begin{array}{l}
x q^{\frac{a+b-1}{2}}, q \sqrt{\cdot},-q \sqrt{\cdot}, q^{\frac{a}{2}}, q^{\frac{a+1}{2}} \\
\sqrt{\cdot},-\sqrt{\cdot}, x q^{\frac{b+1}{2}}, x q^{\frac{b}{2}},-x q^{\frac{b+1}{2}}, \\
\\
-q^{\frac{a}{2}}-q^{\frac{a+1}{2}}, x \\
\quad-x q^{\frac{b}{2}}, q^{\frac{a+b+1}{2}} ; q, x q^{b-a}
\end{array}\right],
\end{array}
\end{aligned}
$$

$\left|x q^{b-a}\right|<1$, whose limit is

$$
\begin{aligned}
F\left(a, b ; \frac{a+b+1}{2} ; x /(x-1)\right) \\
\quad=\left(\frac{1-x}{1+x}\right)^{a} F\left(\frac{a}{2}, \frac{a+1}{2} ; \frac{a+b+1}{2} ; 4 x(1+x)^{-2}\right)
\end{aligned}
$$

that reduces to $[15,2.11(20)]$ when $x$ is replaced by $z /(z-1)$. Application of (2.12) on the ${ }_{8} \phi_{7}$ series on the right side of (3.17) gives two more formulas:

$$
\begin{aligned}
& { }_{2} \phi_{2}\left(q^{a}, q^{b} ; q^{\frac{a+b+1}{2}}, x q^{b} ; q, x q^{\frac{b-a+1}{2}}\right) \\
& =\frac{\left(x^{2} q^{2 b-a}, x^{2} q^{b-a+1} ; q^{2}\right) \frac{a}{2}}{\left(x^{2} q^{b-a} ; q\right)_{a}} \\
& \cdot{ }_{8} \phi_{7}\left[\begin{array}{l}
-x q^{b-1}, q \sqrt{\cdot},-q \sqrt{\cdot}, q^{\frac{a}{2}}, q^{\frac{b}{2}},-q^{\frac{a}{2}}, \\
\sqrt{\cdot},-\sqrt{\cdot},-x q^{b-\frac{a}{2}},-x q^{\frac{b}{2}}, x q^{b-\frac{a}{2}}, \\
-q^{\frac{b}{2}},-x q^{\frac{b-a-1}{2}} \\
x q^{\frac{b}{2}}, q^{\frac{a+b+1}{2}}
\end{array}\right.
\end{aligned}
$$

$\left|x q^{\frac{b-a+1}{2}}\right|<1$, and

$$
\begin{aligned}
& { }_{2} \phi_{2}\left(q^{a}, q^{b} ; q^{\frac{a+b+1}{2}}, x q^{b} ; q, x q^{\frac{b-a+1}{2}}\right) \\
& =\frac{\left(x q^{b+\frac{1-a}{2}}, x q^{\frac{b+1-a}{2}} ; q\right)_{\frac{a-1}{2}}\left(-x q^{b+\frac{1-a}{2}},-x q^{\frac{b-a-1}{2}} ; q\right)_{\frac{a+1}{2}}}{\left(x^{2} q^{b-a} ; q\right)_{a}} \\
& \cdot{ }_{8} \phi_{7}\left[\begin{array}{c}
-x q^{b}, q \sqrt{\cdot},-q \sqrt{\cdot}, q^{\frac{a+1}{2}}, q^{\frac{b+1}{2}} \\
\sqrt{\cdot},-\sqrt{\cdot},-x q^{b+\frac{1-a}{2}},-x q^{\frac{b+1}{2}}, \\
\left.-q^{\frac{a+1}{2}},-q^{\frac{b+1}{2}},-x q^{\frac{b-a+1}{2}} ; q,-x q^{\frac{b-a-1}{2}}\right] \\
x q^{b+\frac{1-a}{2}}, x q^{\frac{b+1}{2}}, q^{\frac{a+b+1}{2}}
\end{array}\right],
\end{aligned}
$$

$\left|x q^{\frac{b-a-1}{2}}\right|<1$. Their limits are

$$
\begin{aligned}
& F\left(a, b ; \frac{a+b+1}{2} ; x /(x-1)\right) \\
& \quad=F\left(\frac{a}{2}, \frac{b}{2} ; \frac{a+b+1}{2} ;-4 x(1-x)^{-2}\right),
\end{aligned}
$$




$$
\begin{aligned}
F( & \left(a, b ; \frac{a+b+1}{2} ; x /(x-1)\right) \\
\quad & =\frac{1+x}{1-x} F\left(\frac{a+1}{2}, \frac{b+1}{2} ; \frac{a+b+1}{2} ;-4 x(1-x)^{-2}\right)
\end{aligned}
$$

which are the same as [15, 2.11(18)] and [15, 2.11(19)], respectively, when $x$ is replaced by $z /(z-1)$.

We now apply (2.11) on the ${ }_{2} \phi_{1}$ series in (3.9) to get

$$
\begin{aligned}
& { }_{8} \phi_{7}\left[\begin{array}{c}
-x q^{2 b-1}, q \sqrt{\cdot},-q \sqrt{\cdot}, q^{a}, q^{b},-q^{a},-q^{b},-x q^{b-a+\frac{1}{2}} \\
\sqrt{\cdot},-\sqrt{\cdot},-x q^{2 b-a},-x q^{b}, x q^{2 b-a}, x q^{b}, q^{a+b-\frac{1}{2}}
\end{array} ; q,-x q^{b-a-\frac{1}{2}}\right] \\
& =\frac{\left(x^{2} q^{2 b-2 a} ; q\right)_{2 a-1}}{\left(x q^{b-a+\frac{1}{2}}, x q^{2 b-a} ; q\right)_{a-1}\left(-x q^{b-a-\frac{1}{2}},-x q^{2 b-a} ; q\right)_{a}} \\
& \quad \cdot{ }_{2} \phi_{2}\left(q^{2 a-1}, q^{2 b-1} ; q^{a+b-\frac{1}{2}}, x q^{2 b-1} ; q, x q^{b-a+\frac{1}{2}}\right), \\
& \left|x q^{b-a-\frac{1}{2}}\right|<1, \text { whose } q \rightarrow 1^{-} \text {limit is } \\
& \begin{array}{c}
\text { (3.24) } \quad F\left(a, b ; a+b-\frac{1}{2} ;-4 x(1-x)^{-2}\right) \\
\quad=\frac{1-x}{1+x} F\left(2 a-1,2 b-1 ; a+b-\frac{1}{2} ; x /(x-1)\right)
\end{array}
\end{aligned}
$$

that reduces to $[15,2.11(13)]$ when $x$ is replaced by $(\sqrt{1-t}-1) /(\sqrt{1-t}+1)$. Use of the same transformation in (3.11) gives

$$
\begin{aligned}
& { }_{8} \phi_{7}\left[\begin{array}{l}
-x q^{2 b-1}, q \sqrt{\cdot},-q \sqrt{\cdot}, q^{a}, q^{b},-q^{a-\frac{1}{2}} \\
\sqrt{\cdot},-\sqrt{\cdot},-x q^{2 b-a},-x q^{b}, x q^{2 b-a+\frac{1}{2}},
\end{array}\right. \\
& \left.=\frac{-q^{b-\frac{1}{2}},-x q^{b-a-\frac{1}{2}}}{x q^{b+\frac{1}{2}}, q^{a+b+\frac{1}{2}}} ; q,-x q^{b-a+\frac{1}{2}}\right] \\
& =\frac{\left(x^{2} q^{2 b-2 a} ; q\right)_{2 a}}{\left(x q^{b-a+\frac{1}{2}}, x q^{2 b-a},-x q^{b-a+\frac{1}{2}},-x q^{2 b-a} ; q\right)_{a}} \\
& \cdot{ }_{2} \phi_{2}\left(q^{2 a}, q^{2 b} ; q^{a+b+\frac{1}{2}}, x q^{2 b} ; q, x q^{b-a+\frac{1}{2}}\right),
\end{aligned}
$$

$\left|x q^{b-a+\frac{1}{2}}\right|<1$, whose $q \rightarrow 1^{-}$limit is

$$
\begin{aligned}
& F\left(a, b ; a+b+\frac{1}{2} ;-4 x(1-x)^{-2}\right) \\
& \quad=F\left(2 a, 2 b ; a+b+\frac{1}{2} ; x /(x-1)\right)
\end{aligned}
$$

which is the same as $[15,2.11(10)]$ if we replace $x$ by $(\sqrt{1-z}-1) /(\sqrt{1-z}+1)$. Observe that (3.25) is also a $q$-analogue of $[15,2.11(2)]$.

We now come to the last three formulas that are derivable from (2.8) via (2.11) and (2.12). First, we replace $b$ by $1+a-c$ in (3.1), then replace $a$ by $c-a$ and finally use (2.11) to get

$$
\begin{aligned}
& { }_{8} \phi_{7}\left[\begin{array}{c}
x q^{c-1}, q \sqrt{\cdot},-q \sqrt{\cdot}, q^{\frac{c-a}{2}}, q^{\frac{c-a+1}{2}} \\
\sqrt{\cdot},-\sqrt{\cdot}, x q^{\frac{c a}{2}}, x q^{\frac{c-a-1}{2}},-x q^{\frac{c+a}{2}},
\end{array}\right. \\
& \begin{array}{l}
-q^{\frac{c-a}{2}},-q^{\frac{c-a+1}{2}}, x \\
\left.\quad-x q^{\frac{c+a-1}{2}}, q^{c} ; q, x q^{2 a-1}\right]
\end{array} \\
& =\frac{\left(x q^{c} ; q\right)_{2 a-c-1}}{\left(x^{2} q^{a+c-1} ; q\right)_{a-c}}{ }_{2} \phi_{2}\left(q^{a}, q^{1-a} ; q^{c}, x q^{a} ; q, x q^{a+c-1}\right) \text {, }
\end{aligned}
$$


$\left|x q^{2 a-1}\right|<1$, whose $q \rightarrow 1^{-}$limit is

$$
\begin{aligned}
& F\left(\frac{c-a}{2}, \frac{c-a+1}{2} ; c ; 4 x(1+x)^{-2}\right) \\
& \quad=(1-x)^{a-1}(1+x)^{c-a} F(a, 1-a ; c ; x /(x-1))
\end{aligned}
$$

which reduces to $[15,2.11(24)]$ when $x$ is replaced by $z /(z-1)$. Applying (2.12) on the ${ }_{8} \phi_{7}$ series in (3.27) in an obvious way we also obtain

$$
\begin{aligned}
{ }_{8} \phi_{7}\left[\begin{array}{c}
-x q^{a+c-2}, q \sqrt{\cdot},-q \sqrt{\cdot}, q^{\frac{c-a}{2}}, q^{\frac{c+a-1}{2}} \\
\sqrt{\cdot},-\sqrt{\cdot},-x q^{\frac{3 a+c-1}{2}},-x q^{\frac{c+a-1}{2}}, q^{c},
\end{array}\right. \\
\quad-x q^{a-1},-q^{\frac{c-a}{2}},-q^{\frac{c+a-1}{c}} \\
\left.x q^{\frac{3 a+c}{2}-1}, x q^{\frac{c+a-1}{2}} ; q,-x q^{a}\right] \\
\quad=\frac{\left(x q^{\frac{c+a}{2}},-x q^{\frac{c+a}{2}} ; q\right)_{a-1}}{\left(-x q^{a} ; q\right)_{c-1}\left(x^{2} q^{c+a-1} ; q\right)_{a-c}}{ }_{2} \phi_{2}\left(q^{a}, q^{1-a} ; q^{c}, x q^{a} ; q, x q^{a+c-1}\right),
\end{aligned}
$$

$\left|x q^{a}\right|<1$, and

$$
\begin{aligned}
& { }_{8} \phi_{7}\left[\begin{array}{c}
-x q^{a+c-1}, q \sqrt{\cdot},-q \sqrt{\cdot}, q^{\frac{c+a}{2}}, q^{\frac{c-a+1}{2}},-q^{\frac{c+a}{2}}, \\
\sqrt{\cdot},-\sqrt{\cdot},-x q^{\frac{c a+a}{2}},-x q^{\frac{c+a-1}{2}}, x q^{\frac{c a}{2}},
\end{array}\right. \\
& \left.-q^{\frac{c+a+1}{2}},-x q^{a} ; q,-x q^{a-1}\right] \\
& =\frac{\left(x q^{\frac{c+a-1}{2}},-x q^{\frac{c+a-1}{2}} ; q\right)_{a}}{\left(-x q^{a-1} ; q\right)_{c+1}\left(x^{2} q^{c+a-1} ; q\right)_{a-c}}{ }_{2} \phi_{2}\left(q^{a}, q^{1-a} ; q^{c}, x q^{a} ; q, x q^{c+a-1}\right),
\end{aligned}
$$

$\left|x q^{a-1}\right|<1$. The limits of (3.29) and (3.30) are, respectively,

$$
\begin{aligned}
& F\left(\frac{c-a}{2}, \frac{c+a-1}{2} ; c ;-4 x(1-x)^{-2}\right) \\
& \quad=(1-x)^{c-1} F(a, 1-a ; c, x /(x-1)),
\end{aligned}
$$

and

$$
\begin{aligned}
& F\left(\frac{c-a+1}{2}, \frac{c+a}{2} ; c ;-4 x(1-x)^{-2}\right) \\
& \quad=(1-x)^{c}(1+x)^{-1} F(a, 1-a ; c ; x /(x-1))
\end{aligned}
$$

which reduce to $[15,2.11(22)]$ and $[15,2.11(23)]$ when $x$ is replaced by $z /(z-1)$. Another $q$-analogue of $[15,2.11(22)]$ different from (3.29) that is valid for terminating series only is given in [19, Ex. (3.3)].

It is clear that the sixteen basic hypergeometric quadratic transformations that we have listed in this section have their origin in (2.8).

\section{QUADRATIC TRANSFORMATION FORMULAS. II}

In this section we shall list all the quadratic transformation formulas that originate from (2.9). Replacing $a, b$ by $q^{a}, q^{b}$, respectively, we can rewrite 
(2.9) in the form

$$
\begin{aligned}
& { }_{2} \phi_{1}\left(q^{2 a}, q^{2 b} ; q^{2+2 a-2 b} ; q^{2}, x^{2} q^{2-4 b}\right) \\
& =\frac{\left(-x q^{\frac{1}{2}-b},-x q^{\frac{3}{2}+a-3 b} ; q\right) a}{\left(x^{2} q^{1-2 b}, x^{2} q^{2-4 b} ; q^{2}\right)_{a}} \\
& \cdot{ }_{8} \phi_{7}\left[\begin{array}{l}
-x q^{\frac{1}{2}+2 a-3 b}, q \sqrt{\cdot},-q \sqrt{\cdot}, q^{a}, q^{\frac{1}{2}+a-b},-q^{\frac{1}{2}+a-b},-q^{1+a-2 b} \\
\sqrt{\cdot},-\sqrt{\cdot},-x q^{\frac{3}{2}+a-3 b},-x q^{1+a-2 b}, x q^{1+a-2 b}, x q^{\frac{1}{2}+a-b}, \\
-x q^{\frac{1}{2}-b} \\
\left.q^{1+2 a-2 b} ; q,-x q^{\frac{1}{2}-b}\right],
\end{array}\right.
\end{aligned}
$$

$\left|x q^{\frac{1}{2}-b}\right|<1$, which approaches the limit

$$
\begin{aligned}
& F\left(a, b ; 1+a-b ; x^{2}\right) \\
& \quad=(1-x)^{-2 a} F\left(a, \frac{1}{2}+a-b ; 1+2 a-2 b ;-4 x(1-x)^{-2}\right)
\end{aligned}
$$

which is the same as $[15,2.11(36)]$ with $x$ replaced by $-z$. Note that $(4.1)$ also gives a $q$-analogue of $[15,2.11(31)]$.

Let us now replace $b$ by $\frac{1+a-b}{2}$ in (4.1) and use (2.11) on the left-hand side. This gives the formula

$$
\begin{aligned}
& { }_{2} \phi_{2}\left(q^{2 a}, q^{2 b} ; q^{a+b+1}, x^{2} q^{2 b} ; q^{2}, x^{2} q^{b-a+1}\right) \\
& =\frac{\left(-x q^{\frac{b-a}{2}},-x q^{\frac{3 b-a}{2}} ; q\right)_{a}}{\left(x^{2} q^{b-a} ; q^{2}\right)_{a}} \\
& \cdot{ }_{8} \phi_{7}\left[\begin{array}{l}
-x q^{\frac{3 b+a}{2}-1}, q \sqrt{\cdot},-q \sqrt{\cdot}, q^{a}, q^{\frac{a+b}{2}}, \\
\sqrt{\cdot},-\sqrt{\cdot},-x q^{\frac{3 b-a}{2}},-x q^{b}, \\
\quad-q^{\frac{a+b}{2}},-q^{b},-x q^{\frac{b-a}{2}} \\
x q^{b}, x q^{\frac{a+b}{2}}, q^{a+b} ; q,-x q^{\frac{b-a}{2}}
\end{array}\right],
\end{aligned}
$$

$\left|x q^{\frac{b-a}{2}}\right|<1$. In the limit $q \rightarrow 1^{-}$this approaches, with $x$ replaced by $-x$,

$$
\begin{aligned}
F\left(a, b ; \frac{a+b+1}{2} ; x^{2} /\left(x^{2}-1\right)\right) \\
\quad=\left(\frac{1-x}{1+x}\right)^{a} F\left(a, \frac{a+b}{2} ; a+b ; 4 x(1+x)^{-2}\right)
\end{aligned}
$$

which is the same as $[15,2.11(21)]$ with $x$ replaced by $(z /(z+1))^{\frac{1}{2}}$.

Next we replace $b$ by $\frac{1}{2}+a-b$ in (4.1), then apply (2.11) on the left-hand side to get

$$
\begin{aligned}
{ }_{8} \phi_{7}\left[\begin{array}{l}
-x q^{3 b-a-1}, q \sqrt{\cdot},-q \sqrt{\cdot}, q^{a}, q^{b},-q^{b}, \\
\sqrt{\cdot},-\sqrt{\cdot},-x q^{3 b-2 a},-x q^{2 b-a}, x q^{2 b-a},
\end{array}\right. \\
-q^{2 b-a},-x q^{b-a} \\
\left.x q^{b}, q^{2 b} ; q-x q^{b-a}\right] \\
=\frac{\left(x^{2} q^{2 b-2 a} ; q^{2}\right)_{a}}{\left(-x q^{b-a},-x q^{3 b-2 a} ; q\right)_{a}} \\
\quad \cdot{ }_{2} \phi_{2}\left(q^{2 a}, q^{4 b-2 a} ; q^{2 b+1}, x^{2} q^{4 b-2 a} ; q^{2}, x^{2} q^{1+2 b-2 a}\right),
\end{aligned}
$$


$\left|x q^{b-a}\right|<1$, whose $q \rightarrow 1^{-}$limit is

$$
\begin{aligned}
& F\left(a, b ; 2 b ;-4 x(1-x)^{-2}\right) \\
& \quad=\left(\frac{1+x}{1-x}\right)^{-a} F\left(a, 2 b-a ; b+\frac{1}{2} ; x^{2} /\left(x^{2}-1\right)\right)
\end{aligned}
$$

which is the same as $[15,2.11(30)]$ with $x$ replaced by $(\sqrt{1-t}-1) /(\sqrt{1-t}+1)$.

The last transformation formula of this section is

$$
\begin{aligned}
& { }_{2} \phi_{2}\left(q^{2 a}, q^{2-2 a} ; q^{2 b}, x^{2} q^{2 a} ; q^{2}, x^{2} q^{2 a+2 b-2}\right) \\
& =\frac{\left(x^{2} q^{2 b-1} ; q^{2}\right)_{a-b}\left(x q^{a-\frac{1}{2}} ; q\right)_{2 b-1}}{\left(x^{2} q^{2 a} ; q^{2}\right)_{b-1}\left(-x q^{b-\frac{1}{2}} ; q\right)_{2 a-1}} \\
& \cdot{ }_{8} \phi_{7}\left[\begin{array}{l}
x q^{2 b+a-\frac{5}{2}}, q \sqrt{\cdot},-q \sqrt{\cdot}, q^{a+b-1}, q^{b-\frac{1}{2}},-q^{b-\frac{1}{2}},-q^{b-a} \\
\sqrt{\cdot},-\sqrt{\cdot}, x q^{b-\frac{1}{2}}, x q^{b+a-1},-x q^{b+a-1},-x q^{b+2 a-\frac{3}{2}}
\end{array}\right. \\
& \left.q^{2 b-1} ; q, x q^{a-\frac{1}{2}}\right]
\end{aligned}
$$

$\left|x q^{a-\frac{1}{2}}\right|<1$, whose $q \rightarrow 1^{-}$limit is

$$
\begin{aligned}
& F\left(a, 1-a ; b ; x^{2} /\left(x^{2}-1\right)\right) \\
& \quad=(1-x)^{a}(1+x)^{2-a-2 b} F\left(a+b-1 ; b-\frac{1}{2} ; 2 b-1 ; 4 x(1+x)^{-2}\right)
\end{aligned}
$$

which reduces to $[15,2.11(25)]$ when we replace $x$ by $(z /(1+z))^{\frac{1}{2}}$. To prove (4.7) we first replace $b$ by $1+a-b$ in (4.1), use (2.11) on the left side, change $a$ to $b-a$, then use $(2.12)$ on the ${ }_{8} \phi_{7}$ series on the right side.

\section{QUADRATIC TRANSFORMATION FORMULAS. III}

The transformation formulas that we shall present in this section are those in which the $q$-analogues of both Gaussian hypergeometric functions are ${ }_{8} \phi_{7}$ series. They originate from the quadratic transformation formula (2.10). The first one is

$$
\begin{aligned}
& { }_{8} \phi_{7}\left[\begin{array}{l}
x q^{3 b-a-1}, q \sqrt{\cdot},-q \sqrt{\cdot}, q^{a}, q^{b},-q^{b},-q^{2 b-a}, x q^{b-a} \\
\sqrt{\cdot},-\sqrt{\cdot}, x q^{3 b-2 a}, x q^{2 b-a},-x q^{2 b-a},-x q^{b}, q^{2 b}
\end{array} ;, x q^{b-a}\right] \\
& =\frac{\left(x^{2} q^{4 b-4 a} ; q^{2}\right)_{a}\left(x^{2} q^{2 b-2 a} ; q\right)_{2 a}}{\left(x^{4} q^{4 b-4 a} ; q^{2}\right)_{a}\left(x q^{b-a}, x q^{3 b-2 a} ; q\right)_{a}} \\
& \cdot{ }_{8} \phi_{7}\left[\begin{array}{l}
x^{2} q^{2 b-1}, q^{2} \sqrt{\cdot},-q^{2} \sqrt{\cdot}, q^{a}, q^{a+1},-q^{a}, \\
\sqrt{\cdot},-\sqrt{\cdot}, x^{2} q^{2 b-a+1}, x^{2} q^{2 b-a},-x^{2} q^{2 b-a+1},
\end{array}\right. \\
& \begin{array}{l}
-q^{a+1}, x^{2} \\
\left.-x^{2} q^{2 b-a}, q^{2 b+1} ; q^{2}, x^{2} q^{4 b-4 a}\right]
\end{array}
\end{aligned}
$$

provided $\left|x q^{b-a}\right|<1$. The $q \rightarrow 1^{-}$limit of this formula is

$$
\begin{aligned}
F\left(a, b ; 2 b ; 4 x(1+x)^{-2}\right) \\
\quad=\frac{(1+x)^{2 a}}{\left(1+x^{2}\right)^{a}} F\left(\frac{a}{2}, \frac{a+1}{2} ; b+\frac{1}{2} ; 4 x^{2}\left(1+x^{2}\right)^{-2}\right),
\end{aligned}
$$

which reduces to $[15,2.11(28)]$ when $x$ is replaced by $(1-\sqrt{1-t}) /(1+\sqrt{1-t})$. 
To prove (5.1) just replace $a, b, c, d$ in (2.10) by $x^{2} q^{2 b-1}, x^{2}, q^{a}$, and $q^{a}$, respectively, and apply (2.12) on the ${ }_{8} \phi_{7}$ series in base $q$. It should be mentioned that a different $q$-analogue of $[15,2.11(28)]$ in terms of a ${ }_{2} \phi_{1}$ and a ${ }_{3} \phi_{1}$ series was given by Jain [23]. See also [19, Ex. (3.2)].

Applying (2.12) on the ${ }_{8} \phi_{7}$ series on the right side of (5.1) we obtain three more variants of this formula:

$$
\begin{gathered}
{ }_{8} \phi_{7}\left[\begin{array}{l}
\left.x q^{3 b-a-1}, q \sqrt{\cdot},-q \sqrt{\cdot}, q^{a}, q^{b},-q^{b},-q^{2 b-a}, x q^{b-a} ; q, x q^{b-a}\right] \\
\sqrt{\cdot}, \sqrt{\cdot}, x q^{3 b-2 a}, x q^{2 b-a},-x q^{2 b-a},-x q^{b}, q^{2 b}
\end{array}\right. \\
=\frac{\left(x^{2} q^{2 b-2 a} ; q^{2}\right)_{a}\left(x^{2} q^{2 b-a} ; q\right)_{2 b-a}}{\left(x q^{b-a}, x q^{3 b-2 a} ; q\right)_{a}\left(-x^{2} q^{2 b-2 a} ; q\right)_{2 b-a}} \\
\cdot{ }_{8} \phi_{7}\left[\begin{array}{l}
x^{2} q^{4 b-2 a-1}, q^{2} \sqrt{\cdot},-q^{2} \sqrt{\cdot}, q^{2 b-a}, q^{2 b-a+1} \\
\sqrt{\cdot},-\sqrt{\cdot}, x^{2} q^{2 b-a+1}, x^{2} q^{2 b-a},-x^{2} q^{4 b-3 a+1}, \\
\left.-q^{a},-q^{a+1}, x^{2} q^{2 b-2 a} ; q^{2}, x^{2} q^{2 b-2 a}\right], \\
-x^{2} q^{4 b-3 a}, q^{2 b+1} \\
=\frac{\left(x^{2} q^{2 b-a}, x^{2} q^{4 b-3 a},-x^{2} q^{4 b-3 a} ; q^{2}\right)_{\frac{a}{2}}}{\left(-x^{2} q^{2 b-2 a} ; q^{2}\right)_{\frac{a}{2}}\left(x q^{b-a}, x q^{3 b-2 a} ; q\right)_{a}}
\end{array}\right.
\end{gathered}
$$

$$
\begin{aligned}
& =\left(1+x^{2} q^{2 b-2 a-1}\right) \frac{\left(x^{2} q^{2 b-a+1}, x^{2} q^{4 b-3 a+1} ; q^{2}\right)_{\frac{a-1}{2}}\left(-x^{2} q^{4 b-3 a+1} ; q^{2}\right)_{\frac{a+1}{2}}}{\left(-x^{2} q^{2 b-2 a} ; q^{2}\right)_{\frac{a+1}{2}}\left(x q^{b-a}, x q^{3 b-2 a} ; q\right)_{a}} \\
& \cdot{ }_{8} \phi_{7}\left[\begin{array}{l}
-x^{2} q^{4 b-2 a}, q^{2} \sqrt{\cdot},-q^{2} \sqrt{\cdot}, q^{a+1}, \\
\sqrt{\cdot},-\sqrt{\cdot},-x^{2} q^{4 b-3 a+1},-x^{2} q^{2 b-2 a+1},
\end{array}\right. \\
& \begin{array}{l}
q^{2 b-a+1},-q^{a+1},-q^{2 b-a+1}, \\
x^{2} q^{4 b-3 a+1}, x^{2} q^{2 b-2 a+1},
\end{array} \\
& \left.\begin{array}{l}
-x^{2} q^{2 b-2 a+1} \\
q^{2 b+1}
\end{array} q^{2},-x^{2} q^{2 b-2 a-1}\right] .
\end{aligned}
$$

Convergence conditions for (5.3) and (5.4) are $\left|x q^{b-a}\right|<1$, but for (5.5) we need $\left|x q^{b-a}\right|<q^{\frac{1}{2}}$. As $q \rightarrow 1^{-}$the limits of these formulas are respectively,

$$
\begin{aligned}
F\left(a, b ; 2 b ; 4 x(1+x)^{-2}\right) & \\
= & (1+x)^{2 b}(1-x)^{2 b-2 a}\left(1+x^{2}\right)^{a-2 b} \\
& \cdot F\left(b-\frac{a}{2}, b-\frac{a}{2}+\frac{1}{2} ; b+\frac{1}{2} ; 4 x^{2}(1+x)^{-2}\right) \\
(5.7)= & \left(\frac{1+x}{1-x}\right)^{a} F\left(\frac{a}{2}, b-\frac{a}{2} ; b+\frac{1}{2} ;-4 x^{2}\left(1-x^{2}\right)^{-2}\right) \\
(5.8)= & \frac{1+x^{2}}{1-x^{2}}\left(\frac{1+x}{1-x}\right)^{a} F\left(\frac{a+1}{2}, b-\frac{a}{2}+\frac{1}{2} ; b+\frac{1}{2} ;-4 x^{2}\left(1-x^{2}\right)^{-2}\right)
\end{aligned}
$$


which reduce to $[15,2.11(29)],[15,2.11(26)]$, and [15, 2.11(27)], respectively, when we replace $x$ by $(1-\sqrt{1-t}) /(1+\sqrt{1-t})$.

$$
\begin{aligned}
& { }_{8} \phi_{7}\left[\begin{array}{l}
x^{2} q^{2 b-2}, q^{2} \sqrt{\cdot},-q^{2} \sqrt{\cdot}, q^{2 a}, q^{2 a+1},-q^{2 a}, \\
\sqrt{\cdot},-\sqrt{\cdot}, x^{2} q^{2 b-2 a}, x^{2} q^{2 b-2 a-1},-x^{2} q^{2 b-2 a},
\end{array}\right. \\
& \begin{array}{l}
-q^{2 a+1}, x^{2} \\
\left.-x^{2} q^{2 b-2 a-1}, q^{2 b} ; q^{2}, x^{2} q^{4 b-8 a-2}\right]
\end{array} \\
& =\frac{\left(x^{4} q^{4 b-8 a-2} ; q^{2}\right)_{2 a}\left(x q^{b-2 a-\frac{1}{2}}, x q^{3 b-4 a-\frac{3}{2}} ; q\right)_{2 a}}{\left(x^{2} q^{2 b-4 a-1}, x^{2} q^{2 b-4 a}, x^{2} q^{4 b-8 a-2} ; q^{2}\right)_{2 a}} \\
& { }_{8} \phi_{7}\left[\begin{array}{l}
x q^{3 b-2 a-\frac{5}{2}}, q \sqrt{\cdot},-q \sqrt{\cdot}, q^{2 a}, q^{b-\frac{1}{2}},-q^{b-\frac{1}{2}},-q^{2 b-2 a-1}, \\
\sqrt{\cdot},-\sqrt{\cdot}, x q^{3 b-4 a-\frac{3}{2}}, x q^{2 b-2 a-1},-x q^{2 b-2 a-1},-x q^{b-\frac{1}{2}},
\end{array}\right.
\end{aligned}
$$

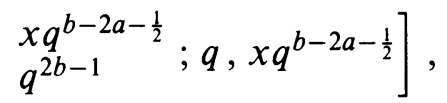

which converges if $\left|x q^{b-2 a-\frac{1}{2}}\right|<1$ and $\left|x q^{2 b-4 a-1}\right|<1$. Its $q \rightarrow 1^{-}$limit is

$$
\begin{aligned}
F\left(a, a+\frac{1}{2} ; b ; 4 x^{2} /\left(1+x^{2}\right)^{-2}\right) \\
\quad=\frac{\left(1+x^{2}\right)^{2 a}}{(1+x)^{4 a}} F\left(2 a, b-\frac{1}{2} ; 2 b-1 ; 4 x(1+x)^{-2}\right),
\end{aligned}
$$

and this reduces to $[15,2.11(17)]$ when $x$ is replaced by $\sqrt{t} /(1+\sqrt{1-t})$.

Let us now replace $a$ and $b$ in (5.3) by $2 a$ and $a+b$, respectively, to get

$$
\begin{aligned}
{ }_{8} \phi_{7}\left[\begin{array}{l}
-x^{2} q^{4 b-2}, q^{2} \sqrt{\cdot},-q^{2} \sqrt{\cdot}, q^{2 a}, q^{2 b},-q^{2 a} \\
\sqrt{\cdot},-\sqrt{\cdot},-x^{2} q^{4 b-2 a},-x^{2} q^{2 b}, x^{2} q^{4 b-2 a},
\end{array}\right. & \left.-q^{2 b},-x^{2} q^{2 b-2 a-1} ; q^{2},-x^{2} q^{2 b-2 a+1}\right] \\
x^{2} q^{2 b}, q^{2 a+2 b+1} \quad & \begin{array}{c}
\left(-x^{2} q^{2 b-2 a} ; q^{2}\right)_{a}\left(x q^{b-a}, x q^{3 b-a} ; q\right)_{2 a} \\
\left(x^{2} q^{2 b}, x^{2} q^{4 b-2 a},-x^{2} q^{4 b-2 a} ; q^{2}\right)_{a}
\end{array} \\
& \cdot{ }_{8} \phi_{7}\left[\begin{array}{c}
x q^{a+3 b-1}, q \sqrt{\cdot},-q \sqrt{\cdot}, q^{2 a}, q^{a+b},-q^{a+b},-q^{2 b}, x q^{b-a} \\
\left.\sqrt{\cdot},-\sqrt{\cdot}, x q^{3 b-a}, x q^{2 b},-x q^{2 b},-x q^{a+b}, q^{2 a+2 b} ; q, x q^{b-a}\right]
\end{array}\right.
\end{aligned}
$$

$\left|x q^{b-a}\right|<1$, whose $q \rightarrow 1^{-}$limit is

$$
\begin{aligned}
F\left(a, b ; a+b+\frac{1}{2} ;-\frac{4 x^{2}}{\left(1-x^{2}\right)^{2}}\right) \\
\quad=\left(\frac{1-x}{1+x}\right)^{2 a} F\left(2 a, a+b ; 2 a+2 b ; \frac{4 x}{(1+x)^{2}}\right)
\end{aligned}
$$

which reduces to $[15,2.11(12)]$ when $x$ is replaced by $\sqrt{z} /(1+\sqrt{1+z})$. Another $q$-analogue of the same formula was given by Jain [23]. See also [19, Ex. (3.2)].

Finally, replacing $a$ and $b$ in (5.5) by $2 a-1$ and $a+b-1$, respectively, 
we get

$$
\begin{aligned}
& { }_{8} \phi_{7}\left[\begin{array}{l}
-x^{2} q^{4 b-2}, q^{2} \sqrt{\cdot},-q^{2} \sqrt{\cdot}, q^{2 a}, q^{2 b},-q^{2 a}, \\
\sqrt{\cdot},-\sqrt{\cdot},-x^{2} q^{4 b-2 a},-x^{2} q^{2 b}, x^{2} q^{4 b-2 a},
\end{array}\right. \\
& \left.\begin{array}{l}
-q^{2 b},-x^{2} q^{2 b-2 a+1} \\
x^{2} q^{2 b}, q^{2 a+2 b-1}
\end{array} q^{2},-x^{2} q^{2 b-2 a-1}\right] \\
& =\frac{\left(-x^{2} q^{2 b-2 a} ; q^{2}\right)_{a}\left(x q^{b-a}, x q^{3 b-a-1} ; q\right)_{2 a-1}}{\left(1+x^{2} q^{2 b-2 a-1}\right)\left(-x^{2} q^{4 b-2 a} ; q^{2}\right)_{a}\left(x^{2} q^{2 b}, x^{2} q^{4 b-2 a} ; q^{2}\right)_{a-1}} \\
& \cdot{ }_{8} \phi_{7}\left[\begin{array}{l}
x q^{a+3 b-3}, q \sqrt{\cdot},-q \sqrt{\cdot}, q^{2 a-1}, q^{a+b-1}, \\
\sqrt{\cdot},-\sqrt{\cdot}, x q^{3 b-a-1}, x q^{2 b-1},-x q^{2 b-1},
\end{array}\right. \\
& \left.\begin{array}{l}
-q^{a+b-1},-q^{2 b-1}, x q^{b-a} \\
-x q^{a+b-1}, q^{2 a+2 b-2}
\end{array} q, x q^{b-a}\right],
\end{aligned}
$$

$\left|x q^{b-a-\frac{1}{2}}\right|<1$, whose $q \rightarrow 1^{-}$limit is

$$
\begin{aligned}
F\left(a, b ; a+b-\frac{1}{2} ;-4 x^{2} /\left(1-x^{2}\right)^{2}\right) \\
=\frac{(1+x)^{2}}{1+x^{2}}\left(\frac{1-x}{1+x}\right)^{2 a} \\
\quad \cdot F\left(2 a-1, a+b-1 ; 2 a+2 b, 4 x /(1+x)^{2}\right)
\end{aligned}
$$

which reduces to $[15,2.11(15)]$ when $x$ is replaced by $\sqrt{z} /(1+\sqrt{1+z})$.

\section{QUADRATIC TRANSFORMATION FORMULAS. IV}

The last group of transformations that we shall present here are meant to be $q$-analogues of the four formulas [15, 2.11(3), (7), (8), (9)] all of whom involve three Gaussian series.

For their derivation we need, in addition to (2.11) and (2.12), the use of Bailey's three-term transformation formulas for a very-well-poised ${ }_{8} \phi_{7}$ series, see $[19,(2.10 .10)$ and $(2.11 .1)]$. For the sake of completeness of this paper and for readers' quick reference we reproduce them here:

$$
\begin{aligned}
&{ }_{8} W_{7}(a ; b, c, d, e, f ; q, \lambda q / b c) \\
&= \frac{(a q, \lambda d / a, \lambda e / a, \lambda f / a ; q)_{\infty}}{(a q / d, q a / e, a q / f, \lambda / a ; q)_{\infty}}{ }_{4} \phi_{3}\left[\begin{array}{l}
d, e, f, a q / b c \\
a q / \lambda, a q / b, a q / c
\end{array} ; q, q\right] \\
&+\frac{(a q, d, e, f, a q / b c, \lambda q / b, \lambda q / c ; q)_{\infty}}{(a q / b, a q / c, a q / d, a q / e, a q / f, \lambda q / b c, a / \lambda ; q)_{\infty}} \\
& \cdot{ }_{4} \phi_{3}\left[\begin{array}{l}
\lambda d / a, \lambda e / a, \lambda f / a, \lambda q / b c \\
\lambda q / a, \lambda q / b, \lambda q / c
\end{array} ;, q\right]
\end{aligned}
$$

where $\lambda=q a^{2} /$ def. The restrictions of this formula are that $|\lambda q / b c|<1$ in the nonterminating case, and that $\lambda / a \neq q^{n}, n=0, \pm 1, \pm 2, \ldots$. The second 
formula is

(6.2)

$$
\begin{aligned}
&{ }_{8} W_{7}(a ; b, c, d, e, f ; q, \lambda q / b c) \\
&=\frac{(a q, b / a, e q / c, f q / c, b e f / a, \lambda d / a, \lambda e / a, \lambda f / a ; q)_{\infty}}{(a q / d, a q / e, a q / f, q / c, e f q / c, \lambda / a, b e / a, b f / a ; q)_{\infty}} \\
& \cdot{ }_{8} W_{7}(e f / c ; a q / b c, a q / c d, e f / a, e, f ; q, b d / a) \\
&+\frac{b}{a} \frac{(a q, b q / a, b q / c, b q / d, b q / e, b q / f, d, e, f, a q / b c, \lambda / b, b q / \lambda ; q)_{\infty}}{\left(a q / b, a q / c, a q / d, a q / e, a q / f, \lambda / a, a q / \lambda, b d / a, b e / a, b f / a, q / c, b^{2} q / a ; q\right)_{\infty}} \\
& \cdot{ }_{8} W_{7}\left(b^{2} / a ; b, b c / a, b d / a, b e / a, b f / a ; q, \lambda q / b c\right),
\end{aligned}
$$

$\lambda=q a^{2} /$ def . The restrictions on the parameters are the same as in $(6.1)$ with the additional condition that $|b d / a|<1$ to ensure convergence of the first ${ }_{8} \phi_{7}$ series on the right side.

By applying (6.1) on the ${ }_{8} \phi_{7}$ series on the left side of (3.25), and using (2.5) and (2.7) we get the following quadratic transformation formula:

$$
\begin{aligned}
& { }_{2} \phi_{2}\left(q^{2 a}, q^{2 b} ; q^{a+b+\frac{1}{2}}, x q^{2 b} ; q, x q^{b-a+\frac{1}{2}}\right) \\
& =\frac{\Gamma_{q}\left(\frac{1}{2}\right) \Gamma_{q}\left(a+b+\frac{1}{2}\right)}{\Gamma_{q}\left(a+\frac{1}{2}\right) \Gamma_{q}\left(b+\frac{1}{2}\right)} \frac{\left(x q^{2 b-a}, x q^{b-a+\frac{1}{2}},-x q^{b-a},-x q^{b-a+\frac{1}{2}} ; q\right)_{a}}{\left(x^{2} q^{2 b-2 a} ; q\right)_{2 a}} \\
& \quad \cdot{ }_{4} \phi_{3}\left[\begin{array}{l}
q^{a}, q^{b},-x q^{b-a},-x q^{b-a-\frac{1}{2}} \\
q^{\frac{1}{2}}, x q^{b}, x q^{2 b-a}
\end{array} q, q\right] \\
& \quad+\frac{\Gamma_{q}\left(-\frac{1}{2}\right) \Gamma_{q}\left(a+b+\frac{1}{2}\right)}{\Gamma_{q}(a) \Gamma_{q}(b)} \\
& \quad \cdot \frac{\left(x q^{2 b-a}, x q^{b-a+\frac{1}{2}},-x q^{b-a},-x q^{b-a+\frac{1}{2}} ; q\right)_{a}\left(1+x q^{b-a-\frac{1}{2}}\right)}{\left(x^{2} q^{2 b-2 a} ; q\right)_{2 a}\left(x q^{b}, x q^{2 b-a} ; q\right)_{\frac{1}{2}}} \\
& \quad \cdot{ }_{4} \phi_{3}\left[\begin{array}{l}
q^{a+\frac{1}{2}}, q^{b+\frac{1}{2}},-x q^{b-a+\frac{1}{2}},-x q^{b-a} \\
q^{\frac{3}{2}}, x q^{b+\frac{1}{2}}, x q^{2 b-a+\frac{1}{2}}
\end{array}, q\right],
\end{aligned}
$$

provided $\operatorname{Re}\left(a+b+\frac{1}{2}\right)>0$. As $q \rightarrow 1^{-}$this approaches the limit

$$
\begin{aligned}
F\left(2 a, 2 b ; a+b+\frac{1}{2} ; x /(x-1)\right) \\
=\frac{\Gamma\left(\frac{1}{2}\right) \Gamma\left(a+b+\frac{1}{2}\right)}{\Gamma\left(a+\frac{1}{2}\right) \Gamma\left(b+\frac{1}{2}\right)} F\left(a, b ; \frac{1}{2} ;\left(\frac{1+x}{1-x}\right)^{2}\right) \\
\quad+\frac{\Gamma\left(-\frac{1}{2}\right) \Gamma\left(a+b+\frac{1}{2}\right)}{\Gamma(a) \Gamma(b)} F\left(a+\frac{1}{2}, b+\frac{1}{2} ; \frac{3}{2} ;\left(\frac{1+x}{1-x}\right)^{2}\right)
\end{aligned}
$$

which reduces to $[15,2.11(3)]$ when $x$ is replaced by $(z+1) /(z-1)$.

To obtain a $q$-analogue of $[15,2.11(7)]$ we proceed as follows. First, from (3.17) we have

$$
\begin{aligned}
{ }_{2} \phi_{2}\left(q^{2 a}, q^{2 b} ; q^{a+b+\frac{1}{2}}, x q^{2 b} ; q, x q^{b+a+\frac{1}{2}}\right) \\
=\frac{\left(x q^{2 b-2 a}, x q^{b-a+\frac{1}{2}} ; q\right)_{2 a}}{\left(x^{2} q^{2 b-2 a} ; q\right)_{2 a}} \\
\quad \cdot{ }_{8} W_{7}\left(x q^{a+b-\frac{1}{2}} ; q^{a}, q^{a+\frac{1}{2}},-q^{a},-q^{a+\frac{1}{2}}, x ; q, x q^{2 b-2 a}\right) .
\end{aligned}
$$


We now apply (6.2) on the ${ }_{8} \phi_{7}$ series on the right side followed by an application of (2.12) on each of the subsequent ${ }_{8} \phi_{7}$ in an obvious manner to obtain the following:

$$
\begin{aligned}
{ }_{8} W_{7}\left(x q^{a+b-\frac{1}{2}} ; q^{a}, q^{a+\frac{1}{2}},-q^{a},-q^{a+\frac{1}{2}}, x ; q, x q^{2 b-2 a}\right) \\
=A_{18} W_{7}\left(q^{a+b+\frac{1}{2}} / x ; q^{b}, q^{b+\frac{1}{2}},-q^{b},-q^{b+\frac{1}{2}}, q / x ; q, q^{1+2 a-2 b} / x\right) \\
\quad+B_{18} W_{7}\left(-q^{b-\frac{1}{2}} ; q^{a}, q^{b},-q^{b},-x q^{b-a-\frac{1}{2}},-q^{a-b+\frac{1}{2}} / x ; q,-q^{1-a}\right),
\end{aligned}
$$

where

$$
\begin{aligned}
A_{1}= & \frac{\left(x q^{a+b+\frac{1}{2}}, x q^{b-a},-x q^{b-a},-x q^{b-a-\frac{1}{2}} ; q\right)_{\infty}}{\left(x q^{b}, x q^{b-2 a-\frac{1}{2}},-x q^{b},-x q^{b+\frac{1}{2}} ; q\right)_{\infty}} \\
& \cdot \frac{\left(q^{a+1} / x, q^{a+\frac{3}{2}} / x, q^{\frac{1}{2}-b} / x, q^{1+2 a-2 b} / x,-q^{a+1} / x,-q^{a+\frac{3}{2}} / x ; q\right)_{\infty}}{\left(q / x, q^{1+a-b} / x, q^{\frac{3}{2}+a+b} / x, q^{2 a-b+\frac{3}{2}} / x,-q^{a-b+\frac{1}{2}} / x,-q^{a-b+1} / x ; q\right)_{\infty}}
\end{aligned}
$$

and

$$
\begin{aligned}
B_{1}= & -\left(1-q^{\frac{1}{2}-b} / x\right) \frac{\left(x q^{a+b+\frac{1}{2}}, x q^{2 b-a}, x^{2} q^{2 b-2 a-1} ; q\right)_{\infty}}{\left(x q^{b-\frac{1}{2}}, x q^{b},-x q^{b},-x q^{b+\frac{1}{2}}, x q^{b-2 a-\frac{1}{2}}, x q^{2 b-2 a} ; q\right)_{\infty}} \\
& \cdot \frac{\left(q^{a+1} / x, q^{2+2 a-2 b} / x^{2} ; q\right)_{\infty}}{\left(q / x, q^{1+a-b} / x, q^{2 a-b+\frac{3}{2}} / x,-q^{\frac{1}{2}+a-b} / x,-q^{1+a-b} / x ; q\right)_{\infty}} \\
& \cdot \frac{\left(q^{\frac{1}{2}},-q^{\frac{1}{2}}, q^{a+\frac{1}{2}},-q^{a+\frac{1}{2}}, q^{b+\frac{1}{2}},-q^{a},-q^{1-a},-q^{\frac{1}{2}+b-a} ; q\right)_{\infty}}{\left(q^{a+b+\frac{1}{2}},-q^{b+\frac{1}{2}} ; q\right)_{\infty}} .
\end{aligned}
$$

For absolute convergence of the three ${ }_{8} \phi_{7}$ series in (6.6) we need the conditions $\left|q^{1-a}\right|<1$ and $\left|q^{2 a-2 b+1}\right|<|x|<\left|q^{2 a-2 b}\right|$.

Note that the first ${ }_{8} \phi_{7}$ series on the right side of (6.6) is the same as that in (6.5) with $a$ and $b$ interchanged and $x$ replaced by $q / x$. So, by using (6.5), (2.5), (2.7) and the identity $\left(q^{\frac{1}{2}},-q^{\frac{1}{2}},-q ; q\right)_{\infty}=1$, we have the desired transformation formula

$$
\begin{aligned}
& \frac{\Gamma_{q}\left(\frac{1}{2}\right) \Gamma_{q}\left(a+b+\frac{1}{2}\right)}{\Gamma_{q}\left(a+\frac{1}{2}\right) \Gamma_{q}\left(b+\frac{1}{2}\right)} \frac{\left(-q^{1-a},-q^{\frac{1}{2}+b-a} ; q\right)_{a}}{\left(-q^{\frac{1}{2}} ; q\right)_{a}(-q ; q)_{a-1}} \frac{q^{\frac{1}{2}-b}}{\left(x+q^{\frac{1}{2}+a-b}\right)} \\
& \frac{\left(x^{2} q^{2 b-2 a-1} ; q\right)_{2 a+1}\left(q^{\frac{3}{2}+a-b} / x ; q\right)_{a}}{\left(q / x, x q^{2 b-2 a} ; q\right)_{a}\left(x q^{b-2 a-\frac{1}{2}} ; q\right)_{3 a+1}} \\
& \cdot{ }_{8} \phi_{8}\left[\begin{array}{l}
-q^{b-\frac{1}{2}}, q \sqrt{\cdot},-q \sqrt{\cdot}, q^{a}, q^{b},-q^{b}-x q^{b-a-\frac{1}{2}}, \\
\sqrt{\cdot},-\sqrt{\cdot},-q^{b-a+\frac{1}{2}},-q^{\frac{1}{2}}, q^{\frac{1}{2}}, q^{a+1} / x,
\end{array}\right. \\
& \left.-q^{a-b+\frac{1}{2}} / x ; q,-q^{1-a}\right] \\
& =\frac{\left(x^{2} q^{2 b-2 a} ; q\right)_{2 a}}{\left(x q^{b-a+\frac{1}{2}}, x q^{2 b-2 a} ; q\right)_{2 a}}{ }_{2} \phi_{2}\left(q^{2 a}, q^{2 b} ; q^{a+b+\frac{1}{2}}, x q^{2 b} ; q, x q^{\frac{1}{2}+b-a}\right) \\
& -\frac{\left(x^{2} q^{2 b-2 a-1}, q^{\frac{1}{2}-b} / x ; q\right)_{2 a+1}}{\left(1+q^{\frac{1}{2}+a-b} / x\right)(q / x ; q)_{2 a}\left(x q^{b-a-\frac{1}{2}}, x q^{b-2 a-\frac{1}{2}} ; q\right)_{2 a+1}} \\
& \text { - }{ }_{2} \phi_{2}\left(q^{2 a}, q^{2 b} ; q^{a+b+\frac{1}{2}}, q^{2 a+1} / x ; q, q^{a-b+\frac{3}{2}} / x\right) \text {, }
\end{aligned}
$$


provided $\operatorname{Re} a<1$. The formal limit of this formula is

(6.10)

$$
\begin{aligned}
& \frac{2 \Gamma\left(\frac{1}{2}\right) \Gamma\left(a+b+\frac{1}{2}\right)}{\Gamma\left(a+\frac{1}{2}\right) \Gamma\left(b+\frac{1}{2}\right)} F\left(a, b ; \frac{1}{2} ;\left(\frac{1+x}{1-x}\right)^{2}\right) \\
& \quad=F\left(2 a, 2 b ; a+b+\frac{1}{2} ; \frac{x}{x-1}\right)+F\left(2 a, 2 b ; a+b+\frac{1}{2} ; \frac{1}{1-x}\right)
\end{aligned}
$$

which reduces to $[15,2.11(7)]$ when $x$ is replaced by $(\sqrt{z}-1) /(\sqrt{z}+1)$. Replacing $b$ by $\frac{1}{2}-b$ in (6.9) and using (2.12) we also find that

$$
\begin{aligned}
& \frac{2 \Gamma_{q}\left(\frac{1}{2}\right) \Gamma_{q}(1+a-b) q^{b}\left(q^{1+a+b} / x ; q\right)_{a}\left(x^{2} q^{-2 a-2 b} ; q\right)_{2 a+1}}{\Gamma_{q}\left(a+\frac{1}{2}\right) \Gamma_{q}(1-b)\left(-1,-q^{\frac{1}{2}} ; q\right)_{a}\left(x+q^{a+b}\right)\left(x q^{-2 a-b} ; q\right)_{3 a+1}} \\
& \cdot{ }_{8} \phi_{7}\left[\begin{array}{c}
x q^{-a-b}, q \sqrt{\cdot},-q \sqrt{\cdot}, q^{a}, q^{b}, x q^{\frac{1}{2}-a-b}, \\
\sqrt{\cdot},-\sqrt{\cdot}, x q^{1-2 a-b}, x q^{1-a-2 b},
\end{array}\right. \\
& \left.-x q^{\frac{1}{2}-a-b},-x q^{-a-b} ; q, q / x\right] \\
& q^{\frac{1}{2}},-q^{\frac{1}{2}},-q \\
& =\frac{\left(x^{2} q^{1-2 a-2 b} ; q\right)_{2 a}}{\left(x q^{1-a-b}, x q^{1-2 a-2 b} ; q\right)_{2 a}}{ }_{2} \phi_{2}\left(q^{2 a}, q^{1-2 b} ; q^{1+a-b}, x q^{1-2 b} ; q, x q^{1-a-b}\right) \\
& -\frac{\left(x^{2} q^{-2 a-2 b}, q^{b} / x ; q\right)_{2 a+1}}{\left(1+q^{a+b} / x\right)(q / x ; q)_{2 a}\left(x q^{-a-b}, x q^{-2 a-b} ; q\right)_{2 a+1}}
\end{aligned}
$$

which is a $q$-analogue of $[15,2.11(8)]$.

For a $q$-analogue of $[15,2.11(9)]$ we first replace $a$ and $b$ in (6.5) by $a-\frac{1}{2}$ and $b-\frac{1}{2}$, respectively, to get

$$
\begin{aligned}
{ }_{2} \phi_{2}\left(q^{2 a-1}, q^{2 b-1} ; q^{a+b-\frac{1}{2}}, x q^{2 b-1} ; q, x q^{b-a+\frac{1}{2}}\right) \\
=\frac{\left(x q^{2 b-2 a}, x q^{b-a+\frac{1}{2}} ; q\right)_{2 a-1}}{\left(x^{2} q^{2 b-2 a} ; q\right)_{2 a-1}} \\
\quad \cdot{ }_{8} W_{7}\left(x q^{a+b+\frac{3}{2}} ; q^{a}, q^{a-\frac{1}{2}},-q^{a},-q^{a-\frac{1}{2}}, x ; q, x q^{2 b-2 a}\right) .
\end{aligned}
$$

Using (6.1) and (2.12) as before we get

$$
\begin{aligned}
{ }_{8} W_{7}\left(x q^{a+b-\frac{3}{2}} ; q^{a}, q^{a-\frac{1}{2}},-q^{a},-q^{a-\frac{1}{2}}, x ; q, x q^{2 b-2 a}\right) \\
\quad=A_{28} W_{7}\left(q^{a+b-\frac{1}{2}} / x ; q^{b}, q^{b-\frac{1}{2}},-q^{b},-q^{b-\frac{1}{2}}, q / x ; q, q^{1+2 a-2 b} / x\right) \\
\quad+B_{28} W_{7}\left(-q^{b+\frac{1}{2}} ; q^{a}, q^{b},-q^{b},-x q^{b-a+\frac{1}{2}},-q^{\frac{3}{2}+a-b} / x ; q,-q^{1-a}\right),
\end{aligned}
$$

where

$$
\begin{aligned}
A_{2}= & \frac{\left(x q^{a+b-\frac{1}{2}}, x q^{b-a},-x q^{b-a},-x q^{b-a+\frac{1}{2}} ; q\right)_{\infty}}{\left(x q^{b}, x q^{b-2 a+\frac{1}{2}},-x q^{b},-x q^{b-\frac{1}{2}} ; q\right)_{\infty}} \\
& \cdot \frac{\left(q^{a+1} / x, q^{a+\frac{1}{2}} / x, q^{\frac{3}{2}-b} / x, q^{1+2 a-2 b} / x,-q^{a+\frac{1}{2}} / x,-q^{a+1} / x ; q\right)_{\infty}}{\left(q / x, q^{1+a-b} / x, q^{a+b+\frac{1}{2}} / x, q^{\frac{1}{2}+2 a-b} / x,-q^{1+a-b} / x,-q^{\frac{3}{2}+a-b} / x ; q\right)_{\infty}},
\end{aligned}
$$


and

$$
\begin{aligned}
B_{2}= & -\left(1-q^{\frac{3}{2}-b} / x\right) \frac{\left(x q^{a+b-\frac{1}{2}}, x q^{2 b-a}, x^{2} q^{2 b-2 a-1} ; q\right)_{\infty}}{\left(x q^{b-\frac{3}{2}}, x q^{b},-x q^{b},-x q^{b-\frac{1}{2}}, x q^{b-2 a+\frac{1}{2}}, x q^{2 b-2 a} ; q\right)_{\infty}} \\
& \cdot \frac{\left(q^{a+1} / x, q^{2+2 a-2 b} / x^{2} ; q\right)_{\infty}}{\left(q / x, q^{1+a-b} / x, q^{\frac{1}{2}+2 a-b} / x,-q^{1+a-b} / x,-q^{\frac{3}{2}+a-b} / x ; q\right)_{\infty}} \\
& \cdot \frac{\left(q^{\frac{3}{2}},-q^{\frac{3}{2}}, q^{a-\frac{1}{2}}, q^{b-\frac{1}{2}},-q^{a},-q^{a-\frac{1}{2}},-q^{1-a},-q^{\frac{3}{2}+b-a} ; q\right)_{\infty}}{\left(q^{a+b-\frac{1}{2}},-q^{b+\frac{3}{2}} ; q\right)_{\infty}}
\end{aligned}
$$

Simplifying the coefficients we find that

$$
\begin{aligned}
& \frac{\Gamma_{q}\left(-\frac{1}{2}\right) \Gamma_{q}\left(a+b-\frac{1}{2}\right)}{\Gamma_{q}\left(a-\frac{1}{2}\right) \Gamma_{q}\left(b-\frac{1}{2}\right)} \frac{\left(-q^{1-a},-q^{\frac{3}{2}+b-a} ; q\right)_{a}}{\left(-q^{-\frac{1}{2}} ; q\right)_{a}(-q ; q)_{a-1}} \frac{q^{\frac{1}{2}-b}}{x} \\
& \quad \cdot \frac{\left(x^{2} q^{2 b-2 a-1} ; q\right)_{2 a}\left(q^{\frac{3}{2}+a-b} / x ; q\right)_{a-1}}{\left(x q^{2 b-2 a} ; q\right)_{a}\left(x q^{b-2 a+\frac{1}{2}} ; q\right)_{3 a-1}(q / x ; q)_{a}} \\
& \quad \cdot{ }_{8} \phi_{7}\left[\begin{array}{l}
-q^{b+\frac{1}{2}}, q \sqrt{\cdot},-q \sqrt{\cdot}, q^{a}, q^{b},-q^{b}, \\
\sqrt{\cdot},-\sqrt{\cdot},-q^{b-a+\frac{3}{2}},-q^{\frac{3}{2}}, q^{\frac{3}{2}},
\end{array}\right. \\
& \left.\quad-x q^{\frac{1}{2}+b-a},-q^{\frac{3}{2}+a-b} / x ; q,-q^{1-a}\right] \\
& =-\frac{\left(x^{2} q^{2 b-2 a} ; q\right)_{2 a-1} \quad q^{a+1} / x, x q^{2 b-a}}{\left(x q^{b-a+\frac{1}{2}}, x q^{2 b-2 a} ; q\right)_{2 a-1}\left(q^{2 a-1}, q^{2 b-1} ; q^{a+b-\frac{1}{2}}, x q^{2 b-1} ; q, x q^{b-a+\frac{1}{2}}\right)} \\
& +\frac{\left(x^{2} q^{2 b-2 a} ; q\right)_{2 a-1}\left(q^{\frac{3}{2}-b} / x ; q\right)_{2 a-1}}{\left(x q^{b-a+\frac{1}{2}}, x q^{b-2 a+\frac{1}{2}}, q / x ; q\right)_{2 a-1}} \\
& \quad \cdot{ }_{2} \phi_{2}\left(q^{2 a-1}, q^{2 b-1} ; q^{a+b-\frac{1}{2}}, q^{2 a} / x ; q, q^{a-b+\frac{3}{2}} / x\right),
\end{aligned}
$$

provided $\operatorname{Re} a<1$. As $q \rightarrow 1^{-}$the formal limit of this formula is

$$
\begin{gathered}
\frac{2 \Gamma\left(-\frac{1}{2}\right) \Gamma\left(a+b-\frac{1}{2}\right)}{\Gamma\left(a-\frac{1}{2}\right) \Gamma\left(b-\frac{1}{2}\right)} \frac{1+x}{1-x} F\left(a, b ; \frac{3}{2} ;\left(\frac{1+x}{1-x}\right)^{2}\right) \\
=F\left(2 a-1,2 b-1 ; a+b-\frac{1}{2} ; \frac{x}{x-1}\right) \\
\quad-F\left(2 a-1,2 b-1 ; a+b-\frac{1}{2} ; \frac{1}{1-x}\right)
\end{gathered}
$$

which reduces to $[15,2.11(9)]$ when $x$ is replaced by $(\sqrt{z}-1) /(\sqrt{z}+1)$.

It may appear that we have left out the formulas (1), (4), and (5) in [15, 2.11]. But the reader will observe that (1) is the same as (32), (4) the same as (28), and (5) the same as (31). So we have exhausted Goursat's list of 36 quadratic transformation formulas in [15].

\section{SOME NEW QUADRATIC TRANSFORMATION FORMULAS}

The title of this section may appear a bit presumptuous but the formulas that we shall derive here do not seem to have appeared elsewhere and may be of some interest to some readers. The idea is extremely simple. If we multiply both sides of Andrews' formula (2.13) by an arbitrary complex sequence $A_{n}$ and sum over $n$ from 0 to $\infty$ assuming, of course, that the series converge on 
both sides, then we get

$$
\begin{gathered}
\sum_{n=0}^{\infty} A_{n} \phi_{3}\left[\begin{array}{l}
q^{-n}, a^{2} q^{n}, b,-b \\
a \sqrt{q},-a \sqrt{q}, b^{2}
\end{array} ;, q\right] \\
=\sum_{n=0}^{\infty} A_{2 n} \frac{\left(q, q a^{2} / b^{2} ; q^{2}\right)_{n}}{\left(q a^{2}, q b^{2} ; q^{2}\right)_{n}} b^{2 n} .
\end{gathered}
$$

A very natural choice of $A_{n}$ would be to render the series on the right side a very-well-poised one. To keep things in manageable limits let us take

$$
A_{n}=\frac{1-a^{2} q^{2 n}}{1-a^{2}} \frac{\left(a^{2}, b^{2}, c, d, e, f ; q\right)_{n}}{\left(q, q a^{2} / b^{2}, q a^{2} / c, q a^{2} / d, q a^{2} / e, q a^{2} / f ; q\right)_{n}}\left(\frac{a^{4} q^{2}}{b^{2} c d e f}\right)^{n}
$$

Then, using [19, (I.25)], we find that

$$
\begin{aligned}
{ }_{12} W_{11} & \left(a^{2} ; b^{2}, c, c q, d, d q, e, e q, f, f q ; q^{2},\left(\frac{a^{4} q^{2}}{b c d e f}\right)^{2}\right) \\
= & \sum_{n=0}^{\infty} \frac{\left(1-a^{2} q^{2 n}\right)\left(a^{2}, b^{2}, c, d, e, f ; q\right)_{n}}{\left(1-a^{2}\right)\left(q, q a^{2} / b^{2}, q a^{2} / c, q a^{2} / d, q a^{2} / e, q a^{2} / f ; q\right)_{n}}\left(\frac{a^{4} q^{2}}{b^{2} c d e f}\right)^{n} \\
& \cdot{ }_{4} \phi_{3}\left[\begin{array}{l}
q^{-n}, a^{2} q^{n}, b,-b \\
a \sqrt{q},-a \sqrt{q}, b^{2}
\end{array} q, q\right] .
\end{aligned}
$$

It the series on either side do not terminate then we ensure convergence by imposing the restrictions $\left|a^{4} q^{2} / b c d e f\right|<1$. The next step is clearly to manipulate the double sum on the right side so that one can derive useful transformation formulas. The big advantage of the ${ }_{4} \phi_{3}$ series on the right is that it is a terminating balanced series, so it can be either transformed to another balanced ${ }_{4} \phi_{3}$ series by Sears' formula [19, (2.10.4)] or to a very-well-poised ${ }_{8} \phi_{7}$ series by Watsons' formula [19, 2.5 .1$)]$. Taking the latter route we have

$$
\begin{aligned}
& { }_{4} \phi_{3}\left[\begin{array}{l}
q^{-n}, a^{2} q^{n}, b,-b \\
a \sqrt{q},-a \sqrt{q}, b^{2}
\end{array} ; q, q\right] \\
& =\frac{\left(b, q a^{2} / b^{2} ; q\right)_{n}}{\left(q a^{2} / b, b^{2} ; q\right)_{n}} b^{n}{ }_{8} \phi_{7}\left[\begin{array}{l}
a^{2} / b, q \sqrt{\bullet},-q \sqrt{\bullet}, a \sqrt{q} / b, \\
\sqrt{\cdot},-\sqrt{\cdot}, a \sqrt{q},-a \sqrt{q}, q a^{2} / b^{2},
\end{array}\right. \\
& \left.\begin{array}{l}
-a \sqrt{q} / b, b, a^{2} q^{n}, q^{-n} \\
q^{1-n} / b, a^{2} q^{n+1} / b
\end{array} ;,-q / b\right] .
\end{aligned}
$$

Substituting this into the right side of (7.3) and interchanging the order of summation (that can be easily justified), we get 


$$
\begin{aligned}
{ }_{12} W_{11} & \left(a^{2} ; b^{2}, c, c q, d, d q, e, e q, f, f q ; q^{2},\left(\frac{a^{4} q^{2}}{b c d e f}\right)^{2}\right) \\
= & \sum_{n=0}^{\infty} \frac{\left(1-\frac{a^{2} q^{2 k}}{b}\right)\left(a^{2} / b, a \sqrt{q} / b,-a \sqrt{q} / b, b ; q\right)_{k}}{\left(1-a^{2} / b\right)\left(q, a \sqrt{q},-a \sqrt{q}, q a^{2} / b^{2} ; q\right)_{k}} \\
& \cdot \frac{(c, d, e, f ; q)_{k}}{\left(q a^{2} / c, q a^{2} / d, q a^{2} / e, q a^{2} / f ; q\right)_{k}} \frac{\left(q a^{2} ; q\right)_{2 k}}{\left(q a^{2} / b ; q\right)_{2 k}}\left(-\frac{a^{4} q^{2}}{b c d e f}\right)^{k} \\
& \cdot{ }_{8} \phi_{7}\left[\begin{array}{c}
a^{2} q^{2 k}, a q^{k+1},-a q^{k+1}, b, c q^{k}, d q^{k}, \\
a q^{k},-a q^{k}, a^{2} q^{2 k+1} / b, a^{2} q^{k+1} / c, a^{2} q^{k+1} / d, \\
e q^{k}, f q^{k} \\
a^{2} q^{k+1} / e, a^{2} q^{k+1} / f
\end{array} ;, \frac{a^{4} q^{2}}{b c d e f}\right] .
\end{aligned}
$$

We shall now point out some special cases of this formula. First, let us force the ${ }_{12} W_{11}$ series on the left and the ${ }_{8} \phi_{7}$ series on the right side to be balanced by taking $q a^{4}=b c d e f$. Then we choose $b=q^{-n}, n=0,1,2, \ldots$, so that we can apply Jackson's ${ }_{8} \phi_{7}$ summation formula $[19,(2.6 .2)]$ to sum the ${ }_{8} \phi_{7}$ series:

$$
\begin{aligned}
& { }_{8} \phi_{7}\left[\begin{array}{l}
a^{2} q^{2 k}, a q^{k+1},-a q^{k+1}, c q^{k}, \\
a q^{k},-a q^{k}, a^{2} q^{k+1} / c, a^{2} q^{k+1} / d,
\end{array}\right. \\
& \left.\begin{array}{l}
d q^{k}, e q^{k}, \frac{a^{4} q^{n+1+k}}{c d e}, q^{-n} \\
a^{2} q^{k+1} / e, \frac{c d e q^{k-n}}{a^{2}}, a^{2} q^{2 k+n+1}
\end{array} ; q, q\right] \\
& =\frac{\left(a^{2} q^{2 k+1}, q a^{2} / c d, q a^{2} / c e, q a^{2} / d e ; q\right)_{n}}{\left(a^{2} q^{k+1} / c, a^{2} q^{k+1} / d, a^{2} q^{k+1} / e, a^{2} q^{1-k} / c d e ; q\right)_{n}} \\
& =\frac{\left(a^{2} q^{2 k+1}, q a^{2} / c d, q a^{2} / c e, q a^{2} / d e ; q\right)_{n}}{\left(a^{2} q^{k+1} / c, a^{2} q^{k+1} / d, a^{2} q^{k+1} / e, q a^{2} / c d e ; q\right)_{n}} \frac{\left(c d e q^{-n} / a^{2} ; q\right)_{k}}{\left(c d e / a^{2} ; q\right)_{k}} \text {. }
\end{aligned}
$$

Using (7.6) in (7.5) we thus obtain the transformation formula:

$$
\begin{aligned}
{ }_{12} W_{11} & \left(a^{2} ; q^{-2 n}, c, c q, d, d q, e, e q, \frac{a^{4} q^{n+1}}{c d e}, \frac{a^{4} q^{n+2}}{c d e} ; q^{2}, q^{2}\right) \\
= & \frac{\left(q a^{2}, q a^{2} / c d, q a^{2} / c e, q a^{2} / d e ; q\right)_{n}}{\left(q a^{2} / c, q a^{2} / d, q a^{2} / e, q a^{2} / c d e ; q\right)_{n}} \\
& \cdot{ }_{10} W_{9}\left(a^{2} q^{n} ; c, d, e, a q^{n+\frac{1}{2}},-a q^{n+\frac{1}{2}}, \frac{a^{4} q^{n+1}}{c d e}, q^{-n} ; q,-q^{n+1}\right) .
\end{aligned}
$$

On the other hand, if we set $f=q^{-n}, b=\frac{a^{4} q^{n+1}}{c d e}$ in (7.5), use [19, (2.6.2)] and then replace $e$ by $a^{4} q^{n+1} / c d e$ we get

$$
\begin{aligned}
{ }_{12} W_{11} & \left(a^{2} ; e^{2}, c, c q, d, d q, \frac{a^{4} q^{n+1}}{c d e}, \frac{a^{4} q^{n+2}}{c d e}, q^{1-n}, q^{-n} ; q^{2}, q^{2}\right) \\
= & \frac{\left(q a^{2}, q a^{2} / c d, q a^{2} / c e, q a^{2} / d e ; q\right)_{n}}{\left(q a^{2} / c, q a^{2} / d, q a^{2} / e, q a^{2} / c d e ; q\right)_{n}} \\
& \cdot{ }_{10} W_{9}\left(a^{2} / e ; a \sqrt{q} / e,-a \sqrt{q} / e, c, d, e, a^{4} q^{n+1} / c d e, q^{-n} ; q,-q / e\right) .
\end{aligned}
$$


We can also derive some other transformation formulas without the balancing condition on the parameters. First use (6.1) on the ${ }_{8} \phi_{7}$ series in (7.5) to get

$$
\begin{aligned}
{ }_{12} W_{11}\left(a^{2} ; b^{2}, c, c q, d, d q, e, e q, f, f q ; q^{2},\left(\frac{a^{4} q^{2}}{b c d e f}\right)^{2}\right) \\
=\sum_{n=0}^{\infty} \frac{\left(1-a^{2} q^{2 k} / b\right)\left(a^{2} / b, a \sqrt{q} / b,-a \sqrt{q} / b, b, f ; q\right)_{k}}{\left(1-a^{2} / b\right)\left(q, a \sqrt{q},-a \sqrt{q}, q a^{2} / b^{2}, q a^{2} / b f ; q\right)_{k}} \\
\quad \cdot\left\{\frac{\left(q a^{2}, q a^{2} / c d, q a^{2} / c e, q a^{2} / d e ; q\right)_{\infty}}{\left(q a^{2} / c, q a^{2} / d, q a^{2} / e, q a^{2} / c d e ; q\right)_{\infty}} \frac{\left(c, d, e, q a^{2} / b f ; q\right)_{k}}{\left(c d e / a^{2}, q a^{2} / f ; q\right)_{k}\left(q a^{2} / b ; q\right)_{2 k}}\right. \\
\quad \cdot q^{k(k+1) / 2}\left(\frac{q a^{2}}{b f}\right)^{2} \phi_{3}\left[\begin{array}{c}
c q^{k}, d q^{k}, e q^{k}, a^{2} q^{k+1} / b f \\
c d e q^{k} / a^{2}, a^{2} q^{k+1} / f, a^{2} q^{2 k+1} / b
\end{array} ;, q\right] \\
\quad+\frac{\left(q a^{2}, c, d, e, q a^{2} / b f, q^{2} a^{4} / c d e f, q^{2} a^{4} / c d e ; q\right)_{\infty}}{\left(q a^{2} / c, q a^{2} / d, q a^{2} / e, q a^{2} / b, q a^{2} / f, c d e / q a^{2}, a^{4} q^{2} / b c d e f ; q\right)_{\infty}} \\
\cdot \frac{\left(c d e / q a^{2} ; q\right)_{k}}{\left(q^{2} a^{4} / b c d e ; q\right)_{k}}\left(-\frac{a^{4} q^{2}}{b c d e f}\right)^{k} \\
\left.\cdot{ }_{4} \phi_{3}\left[\begin{array}{c}
q a^{2} / c d, q a^{2} / c e, q a^{2} / d e, a^{4} q^{2} / b c d e f \\
a^{2} q^{2-k} / c d e, a^{4} q^{k+2} / b c d e, q^{2} a^{4} / c d e f
\end{array} ;, q\right]\right\} .
\end{aligned}
$$

Interchanging the order of summation we obtain

$$
\begin{aligned}
& { }_{12} W_{11}\left(a^{2} ; b^{2}, c, c q, d, d q, e, e q, f, f q ; q^{2},\left(\frac{a^{4} q^{2}}{b c d e f}\right)^{2}\right) \\
& =\frac{\left(q a^{2}, q a^{2} / c d, q a^{2} / c e, q a^{2} / d e ; q\right)_{\infty}}{\left(q a^{2} / c, q a^{2} / d, q a^{2} / e, q a^{2} / c d e ; q\right)_{\infty}} \\
& \cdot \sum_{k=0}^{\infty} \frac{\left(c, d, e, q a^{2} / b f ; q\right)_{k}}{\left(q, c d e / a^{2}, q a^{2} / f, q a^{2} / b ; q\right)_{k}} q^{k} \\
& \cdot{ }_{8} \phi_{7}\left[\begin{array}{c}
a^{2} / b, q \sqrt{\cdot},-q \sqrt{\cdot}, a \sqrt{q} / b,-a \sqrt{q} / b, b, f, q^{-k} \\
\sqrt{\cdot},-\sqrt{\cdot}, a \sqrt{q},-a \sqrt{q}, q a^{2} / b^{2}, q a^{2} / b f, a^{2} q^{k+1} / b
\end{array} ; q,-\frac{a^{2} q^{k+1}}{b f}\right] \\
& +\frac{\left(q a^{2}, c, d, e, q a^{2} / b f, q^{2} a^{4} / b c d e, q^{2} a^{4} / c d e f ; q\right)_{\infty}}{\left(q a^{2} / c, q a^{2} / d, q a^{2} / e, q a^{2} / f, c d e / q a^{2}, a^{4} q^{2} / b c d e f ; q\right)_{\infty}} \\
& \cdot \sum_{k=0}^{\infty} \frac{\left(q a^{2} / c d, q a^{2} / c e, q a^{2} / d e, q^{2} a^{4} / b c d e f ; q\right)_{k}}{\left(q, q^{2} a^{2} / c d e, q^{2} a^{4} / b c d e, q^{2} a^{4} / c d e f ; q\right)_{k}} q^{k} \\
& \cdot{ }_{8} \phi_{7}\left[\begin{array}{l}
a^{2} / b, q \sqrt{\bullet},-q \sqrt{\cdot}, a \sqrt{q} / b,-a \sqrt{q} / b, b, \\
\sqrt{\cdot},-\sqrt{\cdot}, a \sqrt{q},-a \sqrt{q}, q a^{2} / b^{2}, q a^{2} / b f,
\end{array}\right. \\
& \left.\begin{array}{l}
f, c d e q^{-k-1} / a^{2} \\
a^{4} q^{k+1} / b c d e
\end{array} ;,-\frac{a^{4} q^{k+2}}{b c d e f}\right] .
\end{aligned}
$$

In the special case $f= \pm a \sqrt{q}$ both ${ }_{8} \phi_{7}$ series above reduce to ${ }_{6} \phi_{5}$ series which can be summed by the $q$-Dougall formula [19, (2.7.1)]. This leads to the transformation formula 


$$
\begin{aligned}
{ }_{10} W_{9}( & \left.a^{2} ; b^{2}, c, c q, d, d q, e, e q ; q^{2}, \frac{a^{6} q^{3}}{b^{2} c^{2} d^{2} e^{2}}\right) \\
= & \frac{\left(q a^{2}, q a^{2} / c d, q a^{2} / c e, q a^{2} / d e ; q\right)_{\infty}}{\left(q a^{2} / c, q a^{2} / d, q a^{2} / e, q a^{2} / c d e ; q\right)_{\infty}} \\
& \cdot{ }_{5} \phi_{4}\left[\begin{array}{c}
c, d, e, a \sqrt{q} / b,-a \sqrt{q} / b \\
\left.a \sqrt{q},-a \sqrt{q}, q a^{2} / b^{2}, c d e / a^{2} ; q, q\right]
\end{array}\right. \\
& +\frac{\left(q a^{2}, c, d, e, \frac{a^{4} q^{2}}{b^{2} c d e}, \frac{a \sqrt{q}}{b},-\frac{a \sqrt{q}}{b}, \frac{a^{3} q^{\frac{3}{2}}}{c d e},-\frac{a^{3} q^{\frac{3}{2}}}{c d e} ; q\right)_{\infty}}{\left(q a^{2} / b^{2}, q a^{2} / c, q a^{2} / d, q a^{2} / e, c d e / q a^{2}, \frac{a^{3} q^{\frac{3}{2}}}{b c d e},-\frac{a^{2} q^{\frac{3}{2}}}{b c d e}, a \sqrt{q},-a \sqrt{q} ; q\right)_{\infty}} \\
& \left.\cdot{ }_{5} \phi_{4}\left[\begin{array}{c}
q^{2} a^{2} / c d, q a^{2} / c e, q a^{2} / d e, a^{3} q^{\frac{3}{2}} / b c d e,-a^{3} q^{\frac{3}{2}} / b c d e \\
q a^{2} / c d e, a^{4} q^{2} / b^{2} c d e, a^{3} q^{\frac{3}{2}} / c d e,-a^{3} q^{\frac{3}{2}} / c d e
\end{array} q\right], q\right]
\end{aligned}
$$

which is the same as that found by Jain and Verma [27]. If $e=q^{-n}, n=$ $0,1, \ldots$, then the second term on the right side of $(7.11)$ disappears and we get Jain and Verma's [14] transformation formula between a terminating ${ }_{10} \phi_{9}$ in base $q^{2}$ and a terminating balanced ${ }_{5} \phi_{4}$ series in base $q$. Taking the limit $n \rightarrow \infty$ in this formula Bailey's formula [12, (6.3)]. Bailey's second quadratic transformation formula [12, (6.1)] appears to be derivable from (7.1) as well, but it is not. Rather, it is derived by use of the $q$-Saalschütz formula [19, (1.7.2)], as was done in $[19,25$ and 35]. In fact the $n \rightarrow \infty$ limit of [19, (3.10.4)] immediately leads to [12, (6.1)].

Finally, we would like to point out that if we set $b=q^{-n}, n=0,1, \ldots$, in (7.5), then use $[19,(2.5 .1)]$ to reduce the ${ }_{8} \phi_{7}$ series to a terminating balanced ${ }_{4} \phi_{3}$ series then (7.5) takes the form

$$
\begin{aligned}
{ }_{12} W_{11} & \left(a^{2} ; q^{-2 n}, c, c q, d, d q, e, e q, f, f q ; q^{2},\left(\frac{a^{4} q^{n+2}}{c d e f}\right)^{2}\right) \\
= & \frac{\left(q a^{2}, q a^{2} / c d ; q\right)_{n}}{\left(q a^{2} / c, q a^{2} / d ; q\right)_{n}} \sum_{j=0}^{n} \sum_{k=0}^{n} \frac{\left(1-a^{2} q^{2 j+n}\right)\left(a^{2} q^{n}, a q^{n+\frac{1}{2}},-a q^{n+\frac{1}{2}} ; q\right)_{j}}{\left(1-a^{2} q^{n}\right)(q, a \sqrt{q},-a \sqrt{q} ; q)_{j}} \\
& \cdot \frac{\left.(e, f ; q)_{j}\left(q a^{2} / e f, q^{-n} ; q\right)\right]_{k}(c, d ; q)_{j+k}}{\left(a^{2} q^{n+1} / c, a^{2} q^{n+1} / d ; q\right)_{j}\left(q, c d q^{-n} / a^{2} ; q\right)_{k}\left(q a^{2} / e, q a^{2} / f ; q\right)_{j+k}}\left(-\frac{a^{4} q^{n+2}}{c d e f}\right)^{j} q^{k}
\end{aligned}
$$

Letting $c, d, e, f$ and $n \rightarrow \infty$ in this formula we obtain

$$
\begin{aligned}
& 1+\sum_{j=1}^{\infty}\left(1-a^{2} q^{4 j}\right) \frac{\left(a^{2} q^{2} ; q^{2}\right)_{j-1}}{\left(q^{2} ; q^{2}\right)_{j}}(-1)^{j} a^{8 j} q^{9 j^{2}-j} \\
& \quad=\left(q a^{2} ; q\right)_{\infty} \sum_{k=0}^{\infty} \frac{q^{k^{2}} a^{2 k}}{(q ; q)_{k}} \sum_{j=0}^{\infty} \frac{q^{\frac{5 j^{2}}{2}}\left(a^{4} q^{2 k-\frac{1}{2}}\right)^{j}}{(q ; q)_{j}\left(q a^{2} ; q^{2}\right)_{j}} .
\end{aligned}
$$

If $a=1$ then the left-hand side becomes

$$
\begin{aligned}
& 1+\sum_{j=1}^{\infty}\left(1+q^{2 j}\right)(-1)^{j} q^{9 j^{2}-j} \\
& \quad=\sum_{j=-\infty}^{\infty}(-1)^{j} q^{9 j^{2}+j}=\left(q^{8}, q^{10}, q^{18} ; q^{18}\right)_{\infty}, \quad \text { by }[19,(1.6 .1)] .
\end{aligned}
$$


We thus have a Rogers-Ramanujan type identity

$$
\sum_{k=0}^{\infty} \frac{q^{k^{2}}}{(q ; q)_{k}} \sum_{j=0}^{\infty} \frac{q^{\frac{5 j^{2}}{2}+\left(2 k-\frac{1}{2}\right) j}}{(q ; q)_{j}\left(q ; q^{2}\right)_{j}}=\frac{\left(q^{8}, q^{10}, q^{18} ; q^{18}\right)_{\infty}}{(q ; q)_{\infty}}
$$

\section{REFERENCES}

1. R. P. Agarwal, Generalized hypergeometric series, Asia Publishing House, Bombay, London and New York, 1963.

2. W. A. Al-Salam and A. Verma, On quadratic transformations of basic series, SIAM J. Math. Anal. 15 (1984), 414-420.

3. G. E. Andrews, On the q-analogue of Kummer's theorem and applications, Duke Math. J. 40 (1973), 525-528.

4. __ Problems and prospects for basic hypergeometric functions, Theory and Applications of Special Functions (R. Askey, ed.), Academic Press, New York, 1975, 191-224.

5. $\ldots$ On q-analogues of the Watson and Whipple summations, SIAM J. Math. Anal. 7 (1976), 332-336.

6. G. E. Andrews and R. Askey, Enumeration of partitions: the role of Eulerian series and q-orthogonal polynomials, Higher Combinatorics (M. Aigner, ed.), Reidel, Boston, Mass., 1977, pp. 3-26.

7. __ Another q-extension of the beta function, Proc. Amer. Math. Soc. 81 (1981), 97-100.

8. R. Askey and J. A. Wilson, Some basic hypergeometric polynomials that generalize Jacobi polynomials, Mem. Amer. Math. Soc., no. 319, 1985.

9. W. N. Bailey, Transformations of generalized hypergeometric series, Proc. London Math. Soc. (2) 29 (1929), 495-502.

10. __ Some identities involving generalized hypergeometric series, Proc. London Math. Soc. (2) 29 (1929), 503-516.

11. _ Generalized hypergeometric series, Cambridge Univ. Press, Cambridge, 1935, reprinted by Stechert-Hafner, New York, 1964.

12. __ Identities of the Rogers-Ramanujan type, London Math. Soc. (2) 50 (1949), 1-10.

13. L. Carlitz, Some formulas of $F$. H. Jackson, Monatsh. Math. 73 (1969), 193-198.

14. V. G. Drinfel'd, Quantum groups, Proceedings of the International Congress of Mathematics, Berkeley, 1986.

15. A. Erdélyi, (ed.), Higher transcendental functions, vol. I, McGraw-Hill, New York, 1953.

16. G. Gasper, Rogers' linearization formula for the continuous q-ultraspherical polynomials and quadratic transformation formulas, SIAM J. Math. Anal. 16 (1985), 1061-1071.

17. G. Gasper and M. Rahman, Product formulas of Watson, Bailey and Bateman types and positivity of the Poisson kernel for q-Racah polynomials, SIAM J. Math. Anal. 15 (1984), 768-789.

18. __ Positivity of the Poisson kernel for the continuous q-Jacobi polynomials and some quadratic transformation formulas for basic hypergeometric series, SIAM J. Math. Anal. 17 (1986), 970-999.

19. __ Basic hypergeometric series, Encyclopedia of Mathematics and its Applications, vol. 35, Cambridge Univ. Press, Cambridge, 1990.

20. C. F. Gauss, Disquisitiones generales circa seriem infinitam $1+\alpha \beta x / 1 . \gamma+\alpha(\alpha+1) \beta(\beta+$ 1) $x^{2} / 1.2 \gamma(\gamma+1)+\cdots$, Comm. Soc. Reg. Sci. Gött. Rec., 1813, Vol. II; reprinted in Werke 3 (1876), 123-162.

21. __ Nachlass, Determinato seriei nostrae per aequationem differentialem secundi ordinis, Werke 3 (1876), 207-229. 
22. Edouard Goursat, Sur l'équation différentielle linéaire, Ann. Sci. Ećole Norm. Sup. (2) 10 (1881), 3-142.

23. V. K. Jain, Some transformations of basic hypergeometric functions, Part II, SIAM J. Math. Anal. 12 (1981), 957-961.

24. _ Certain transformations of basic hypergeometric series and their applications, Pacific J. Math. 101 (1982), 333-349.

25. V. K. Jain and A. Verma, Transformations between basic hypergeometric series on different bases and identities of Rogers-Ramanujan type, J. Math. Anal. Appl. 26 (1980), 230-269.

26. __ Some transformations of basic hypergeometric functions, Part I, SIAM J. Math. Anal. 12 (1981), 943-956.

27. __ Transformations of nonterminating basic hypergeometric series, their contour integrals and applications to Rogers-Ramanujan identities, J. Math. Anal. Appl. 87 (1982), 9-44.

28. T. H. Koornwinder, Representations of the twisted $S U(2)$ quantum group and some $q$ hypergeometric orthogonal polynomials, Nederl. Akad. Wetensch. Proc. Ser. A 92 (1989), 97-117.

29. __ Orthogonal polynomials in connection with quantum groups, Orthogonal Polynomials: Theory and Practice (P. Nevai, ed.), NATO ASI Series C \#294, Kluwer, 1990, pp. 257-292.

30. __ A second addition formula for continuous q-ultraspherical polynomials motivated by quantum groups (to appear).

31. E. Kummer, Über die hypergeometrische Reihe $1+(\alpha \cdot \beta) x / 1 . \gamma+\cdots$, J. Reine Angew. Math. 15 (1836), 39-83 and 127-172, reprinted in Collected Papers (A. Weil, ed.), SpringerVerlag, Berlin and New York, vol. 2, 1975, pp. 75-172.

32. T. Masuda, K. Mimachi, Y. Nakagami, M. Noumi, and K. Ueno, Representations of quantum groups and a q-analogue of orthogonal polynomials, CR Acad. Sci. Paris Sér. I Math. 307 (1988), 559-564.

33. K. Mimachi and M. Noumi, Quantum 2-spheres and big q-Jacobi polynomials, Comm. Math. Phys. 128 (1990), 521-531.

34. B. Nassrallah, Some quadratic transformations and projection formulas for basic hypergeometric series, Thesis, Carleton Univ., Ottawa, 1982.

35. B. Nassrallah and M. Rahman, On q-analogues of some transformations of nearly-poised hypergeometric series, Trans. Amer. Math. Soc. 268 (1981), 211-229.

36. M. Rahman, The linearization of the product of continuous q-Jacobi polynomials, Canad. J. Math. 33 (1981), 255-284.

37. M. Rahman and A. Verma, Product and addition formulas for the continuous q-ultraspherical polynomials, SIAM J. Math. Anal. 17 (1986), 1461-1474.

38. D. B. Sears, Transformations of basic hypergeometric functions of special type, Proc. London Math. Soc. (2) 52 (1951), 467-483.

39. V. N. Singh, The basic analogues of identities of the Cayley-Orr type, J. London Math. Soc. 34 (1959), 15-22.

40. L. J. Slater, Generalized hypergeometric functions, Cambridge Univ. Press, Cambridge, 1966.

41. L. L. Vaksman and Ya. S. Soibel'man, Algebra of functions on the quantum group $S U(2)$, Functional Anal. Appl. 22 (1988), 170-181.

42. N. Ya. Vilenkin, Special functions and the theory of group representations, Transl. Math. Monographs, vol. 22, Amer. Math. Soc., Providence, R.I., 1968.

43. F. J. W. Whipple, Some transformations of generalized hypergeometric series, Proc. London Math. Soc. (2) 26 (1927), 257-272.

44. _ Algebraic proofs of the theorems of Cayley and Orr concerning the products of certain hypergeometric series, J. London Math. Soc. 2 (1927), 85-90.

45. __ On a formula implied in Orr's theorems concerning the product of hypergeometric series, J. London Math. Soc. 4 (1929), 48-50. 
46. S. L. Woronowicz, Compact matrix pseudogroups, Comm. Math. Phys. 111 (1987), 613665 .

47. _ Res. Inst. Math. Sci. 23 (1987), 117-181.

Department of Mathematics and Statistics, Carleton University, Ottawa, Ontario, CANADA K1S 5B6

E-mail address: mizan-rahman@carleton.ca

DePartment of MAthematics, UNIVERsity of RoORKeE, ROORKEe, INDIA 247667 